\title{
A review of recent studies on coronal dynamics: Streamers, coronal mass ejections, and their interactions
}

\author{
CHEN Yao \\ Institute of Space Sciences and School of Space Science and Physics, Shandong University, Weihai 264209, China
}

Received October 8, 2012; accepted December 25, 2012; published online March 26, 2013

\begin{abstract}
In this article I present a review of recent studies on coronal dynamics, including research progresses on the physics of coronal streamers that are the largest structure in the corona, physics of coronal mass ejections (CMEs) that may cause a global disturbance to the corona, as well as physics of CME-streamer interactions. The following topics will be discussed in depth: (1) acceleration of the slow wind flowing around the streamer considering the effect of magnetic flux tube curvature; (2) physical mechanism accounting for persistent releases of streamer blobs and diagnostic results on the temporal variability of the slow wind speed with such events; (3) force balance analysis and energy release mechanism of CMEs with a flux rope magnetohydrodynamic model; (4) statistical studies on magnetic islands along the coronal-ray structure behind a CME and the first observation of magnetic island coalescence with associated electron acceleration; and (5) white light and radio manifestations of CME-streamer interactions. These studies shed new light on the physics of coronal streamers, the acceleration of the slow wind, the physics of solar eruptions, the physics of magnetic reconnection and associated electron acceleration, the large-scale coronal wave phenomenon, as well as the physics accounting for CME shock-induced type II radio bursts.
\end{abstract}

solar wind, coronal streamer, coronal mass ejection, solar radio burst, magnetic reconnection

Citation: Chen Y. A review of recent studies on coronal dynamics: Streamers, coronal mass ejections, and their interactions. Chin Sci Bull, 2013, 58: 1599-1624, doi: $10.1007 / \mathrm{s} 11434-013-5669-6$

The corona is the outermost layer of the solar atmosphere. It has a very high temperature ( million kelvin) consisting of fully-ionized plasmas with electrons, protons, alpha particles and minor heavy ions at various charge states. It is not observable to naked eyes or traditional telescopes because the coronal emission intensity at the visible light is extremely weak, as low as a millionth of the emission intensity from the photosphere. It is visible when the bright photosphere is blocked entirely by the moon during a total solar eclipse.

To study the corona in a routine manner, Lyot invented a special optical telescope called as coronagraph in the 1930s, which creates an artificial total solar eclipse with a disk occulting the bright solar surface. This allows coronal observations on a daily basis. Figure 1(a) presents a coronal image obtained by the LASCO (Large Angle and Spectrometric Coronagraph [1]) $\mathrm{C} 2$ coronagraph on board the SOHO (So-

email: yaochen@sdu.edu.cn lar and Heliospheric Observatory) spacecraft on 2004 July 6, which shows several bright streamers. In Figure 1(b) and (c), we present the coronal magnetic configuration as calculated with the extrapolation of the photospheric magnetic field measurement with the Potential Field Source Surface Model (PFSS: [2,3]) and the schematic of the magnetic configuration of a typical helmet streamer. The good correspondence between the large scale closed magnetic field lines and the bright streamer structures can be told by comparing the three panels. Physically, this reflects the dominant role played by the magnetic field in the formation and evolution of large-scale coronal structures.

The large-scale coronal magnetic structures can be simply divided into two groups, open structures and closed structures. The former mainly correspond to coronal holes that are the major source of the fast solar wind while the later to coronal loops and helmet streamers. According to coronagraph observations, the helmet streamer usually converges 


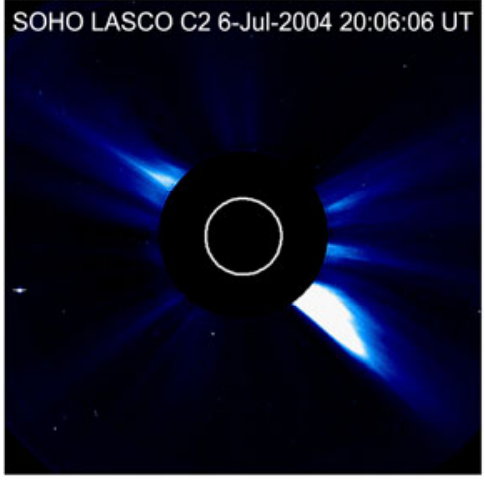

(a)

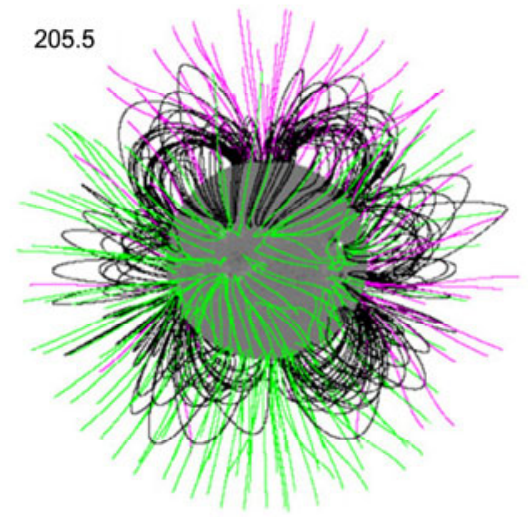

(b)

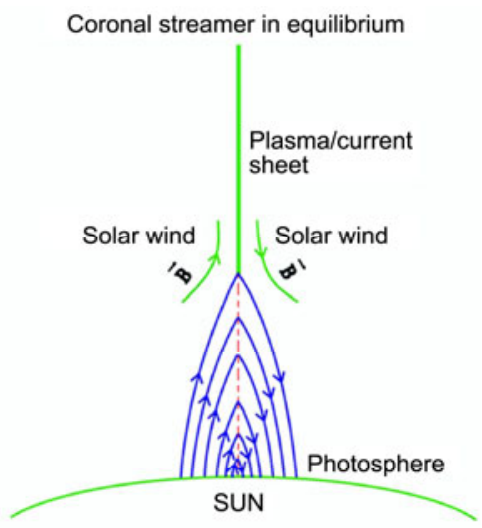

(c)

Figure 1 (a) A coronal image with several bright streamers observed by LASCO C2 coronagraph on July 62004 , the inner white circle represents the solar photosphere and the black disk represents the coronagraph occultor; (b) the magnetic field configuration as given by the PFSS model with the purple (green) lines denoting outward (inward)-directing open field lines, and the black ones are closed field lines; (c) a schematic drawing of the magnetic configuration of a typical streamer, the green arrows denote the direction of the magnetic field, the vertical green line atop the streamer represents the HCS, and the red dashed line gives the center of the streamer structure. Comparing these panels, we can tell the important influence of magnetic field on large-scale coronal structures.

to a cusp structure below $\sim 2-3$ Rs. The streamer cusp is the localized region on the top of the magnetically-closed arcades, and below the bright plasma sheet structure (also called the streamer stalk). The heliospheric current sheet (HCS) is embedded within the plasma sheet [4,5]. Below the cusp, the streamer is bright due to the presence of denser-than-surrounding plasmas confined by its closed fields; while above the cusp the streamer stalk is bright because of the presence of the dense solar wind plasmas there. The streamer region is believed to be an important source of the slow solar wind [6-10]. It has been shown that the streamer magnetic configurations are important to the dynamics of the associated solar wind [11-15]. More discussion on this topic will be presented in the following section.

Streamers are quiescent coronal structures which can persist for a solar rotation or even longer. They frequently exhibit various activites observable with coronagraphs. For instance, coronal materials or density structures carried by the solar wind are overwhelmingly observed to flow outward along the plasma sheet; sometimes one observes one or several blob structures released from the streamer tip $[10,16]$. Also the overall streamer morphology may evolve with time, showing diffusion, gradual expansion, disruption and reformation, and plasma sheet disconnection [17]. It is known that these morphology changes are related to the dynamical coupling of plasmas and magnetic fields, and also related to the projection effect as well as solar rotation. In this paper we will get deeply involved into the subject of streamer activities.

Streamers are closely related to coronal mass ejections (CMEs) that present the largest and strongest energy releases in the solar system. According to previous studies, $~ 60 \%-$ $70 \%$ of CMEs are related to streamers $[18,19]$. The eruptions disrupting the whole streamer structure are dubbed the blowout CME [20,21], and those originate from one foot of a streamer causing partial streamer disruption are sometimes called as puff-CME [22].

It is well known that CMEs release a dramatic amount of coronal energies and masses. In large events, CMEs can disturb the corona and near-Earth space environment on a global scale. They are the major driver of catastrophic space weather events [23]. To forcast the occurrence of these events and their space weather consequences, one needs to understand the underlying mechanism, as well as physics accounting for various accompanying phemomena.

A key issue is to understand the nature of the pre-eruption energy, and how the energy gets accumulated and released eruptively. It is generally believed that the eruption is energized by the coronal magnetic field. The magnetic energy is slowly accumulated in the corona via twisting, shear, and emergence motions of photospheric magnetic fields as a result of the sub-photospheric convections. Under certain conditions, the accumulated magnetic energy gets released eruptively and converted into dynamic and thermal energies of plasmas [24,25]. There are two major types of solar eruptions, flares and CMEs. To flares, the energy is released mainly through magnetic reconnections [26]; to CMEs, however, it is less clear about how energies are released [27-29]. In this paper, some studies on this topic will be presented.

As the laregest eruption in the corona, CMEs usually arouse strong disturbance to the corona and produce various accompanying phenomena, like EUV waves [30,31], various types of radio bursts [32], coronal shocks [33,34], solar energetic particles [35], etc. These processes are different manifestations of solar eruptions, composing a significant part of studies on coronal dynamics. Here of particular interests are interactions of CMEs and streamers. Streamers are frequently located above active regions being a major 
source as well as energy storage agent of solar eruptions. On the other hand, CMEs often manifest as streamer eruptions, especially during solar minimum conditions. Good examples are blowout and puff CMEs [20-22]. CMEs also interact with streamers in other different ways. For instance, CMEs may cause streamer deflections that have been used to trace coronal shocks and diagnose the shock speeds $[33,36]$, and drive reconnections along the streamer current sheet forming blobs [37] or streamer disconnections [17].

In the following sections, I will review some recent studies on streamer properties, acceleration of relevant solar winds, energy release mechanisms of CMEs, reconnection processes along the current sheet behind CMEs, and CMEstreamer interaction processes.

\section{Studies on coronal streamers and associated slow solar wind}

Streamers are large-scale quiescent bright structures in the corona. They are the product of the dynamic equilibrium between confining magnetic fields and expanding hot plasmas [4,5]. Previous studies indicate that streamers and nearby regions are important sources of the slow solar wind [8-10]. The streamer magnetic topologies, including the cusp structure, the co-existence of open and closed fields at the streamer boundary, and the expansion of relevant flux tubes, have deep impacts on the acceleration of the slow wind $[13,38]$. Coronagraph observations reveal the presence of discrete outflowing blob structures atop streamers, which are slightly brighter than the background and regarded as velocity tracers of the slow wind along the plasma sheet $[10,16]$. In the following text, I will introduce lastest studies on ultraviolet (UV) and white light morphologies of streamers, the acceleration process of the streamer-related solar wind, and physics accounting for streamer blobs and relevant velocity diagnostics.

\subsection{Morphological studies on coronal streamers in the white-light and UV wavelengths}

Coronagraphs, as represented by LASCO C2, play a dominant role in the study of streamer morphologies. Due to a lack of simultaneous measurements of coronal magnetic field, there exist different understandings of the magnetic topology underlying white light streamers. It is generally believed that streamers consist of large-scale closed magnetic arcades [4]. However, observations of the past decade indicate that streamers may have more complex magnetic fields. For example, the LASCO $\mathrm{C} 1$ data with the emission line of Fe XIV $530 \mathrm{~nm}$ at the streamer base show a few separate co-existing loop systems [39], implying that multipolar magnetic components may contribute even at solar minimum [40].

The presence of multi-polar components within stream- ers affects dynamics and properties of the associated interplentary structures and solar wind plasmas. For instance, it has been suggested that the heliospheric plasma sheet may contain multi-current sheet structures [41]. And multi-directional discontinuities are indeed observed at the boundary of interplanetary sectors [42]. It also affects the development of the dynamic equilibrium and instabilities of coronal streamers, especially at the cusp region [43]. Furthermore, this indicates that open structures may exist within the streamer. These open structures may have connections to the heliospheric plasma sheet, and serve as a separate origin of the solar wind. It remains unclear whether this source of the solar wind exists, and if it does, how the wind differs from others [44].

From the $\mathrm{pB}$ (polarization Brightness) measurements of coronagraphs, it is seen that dense plasmas are present within the helmet-cusp streamer structure. This is one major observational basis for suggesting streamers being bipolar-closed magnetic structures. Nevertheless, this assumption is not as robust as expected according to a recent coronal and solar wind model driven by Alfvénic turbulence [45]. This indicates that an electron density distribution forming the observed helmet-like $\mathrm{pB}$ structure can be reproduced using a prescribed spatial distribution of turbulence and boundary conditions, even under a completely-open field configuration.

Morphological studies on streamers using the UVCS OVI $1032 \AA$ spectral data reveal a surprising fork-like structure with the core of the streamer darker than its surroundings, while in the Ly-alpha line the streamer presents a typical helmet morphology [46]. It is further found that the OVI fork-like feature is common to many quiescent streamers at solar minimum [47]. Velocity diagnostics in the streamer region using the Doppler dimming technique reveal that the OVI velocity decreases rapidly across the $\mathrm{pB}$ streamer boundary and there is no observable outflow of OVI ions within the streamer core [48]. This seems to be inconsistent with the idea that there exist open field regions inside the streamer. From this respect, the UV study supports the traditional view of streamers below the cusp being closed as a whole. This is actually a working assumption of the few multi-fluid numerical models aimed at understanding the UV streamers [15,49].

The rather sophisticated model by Li et al. [15] indicates that the fork structure observed by UVCS can be understood by tracing the temporal evolution of minor ion distribution, which can be separated into two phases. The first one corresponds to the stabilizing process of the streamer. The evolving time scale is determined by the Alfvénic transit time with a typical value of several tens of hours. In this process there exists a significant amount of plasma exchange between open and closed regions due to the ion motion across field lines, and minor ions may flow into the upper region of the streamer core. In the second phase, minor ions settle down along the field lines due to gravity. This explains the lack of OVI emission in the streamer core as ob- 
served by UVCS. Due to strong Coulomb collisions between protons and minor ions, the gravitational settling process of minor ions is rather slow with a settling speed of about $1-2 \mathrm{~km} \mathrm{~s}^{-1}$ and a time scale of $80 \mathrm{~h}$. The model predicts that the OVI fork-like feature appears several days after the stabilization of a devolping streamer. This has yet to be tested observationally, though.

\subsection{Acceleration of the slow solar wind associated with coronal streamers}

Under solar minimum conditions, the solar wind can be simply divided into two components, the fast wind at high latitudes and the slow wind at low latitudes [50,51]. Although extensive studies on the solar wind acceleration have been conducted in the past half century, it remains elusive regarding why the fast wind is fast and slow wind slow. It is known that the acceleration of the solar wind is closely related to its origin. There exist two competing scenarios accounting for solar wind origins and accelerations. The first one claims that the solar wind plasmas are mainly energized by waves or turbulence propagating outwards from the Sun; the second one claims that reconnections between open and closed magnetic fields in the lower solar atmosphere are important for the mass and energy supply to the solar wind [52].

Here we focus on the former wave-driven scenario. With this assumption, the solar wind streams outwards along a flux tube rooted on the photosphere. The low-frequency waves that are driven by sub-photospheric convection heat the solar wind plasmas through wave-particle interaction, and the solar wind is accelerated by the gradient forces of thermal pressure and wave pressure. Both fast and slow winds can be explained with the same mechanism. Different properties of the solar winds may simply result from different boundary conditions as well as different flux tube geometries [13,53].

Observationally, the solar wind speeds as measured insitu are found to correlate with the expansion rate of the flux tube in the corona [54] and the angular distance from the flux tube footpoints to nearby coronal holes [55]. This provides support to the wave-driven scenario of the solar wind origin. A natural question to ask is that how the solar wind parameters depend on the flux tube geometry or in other words, under what kind of tube geometry the solar wind is slow (or fast)?

The tube geometry surrounding streamers is stongly affected by the streamer. As a result, these flux tubes often present a special expansion-convergence and re-expansion process with an apparent curvature along the streamer boundary. Effect of the tube expansion on the slow wind acceleration has been extensively examined in previous studies $[13,53,56]$, while the effect of the flux tube curvature was considered only recently [57] with a one-dimensional (1-D) electron-proton solar wind model driven by Alfvénic turbulence. The field configuration is taken from the analytic model of the coronal magnetic field given by Banaszkiewicz et al. [40]. Li et al. [45] found that both the expansion and curvature of the flux tube contribute considerable to the solar wind dynamics.

In Figure 2 variations of the solar wind speed at $1 \mathrm{AU}$ for two cases are shown, with Case $\mathrm{C}$ considering the tube curvature while Case $\mathrm{S}$ not. It can be seen that in both cases the solar wind speed at $1 \mathrm{AU}$ strongly anti-correlates with the flux tube expansion rate in the corona. And the slow wind speed is considerably lower in Case $\mathrm{C}$ than in Case S. The relative speed difference reaches a maximum value of $\sim 30 \%$ at the equator, as a result of the tube curvature. This result can be understood from the spatial distribution of the momentum and energy depositions below and beyond the sonic point [58]. If the sonic points in both cases are at the same distance, more energy/momentum will be deposited into the sub-sonic region in Case $\mathrm{C}$ than in Case $\mathrm{S}$ due to the curvature effect. This results in a higher mass flux and a slower solar wind in Case C.

Further studies indicate that the above curvature effect on the solar wind speed holds for more general boundary conditions, different heating mechanisms, and presence of alpha particles [59]. Another relevant observation reveals that filamentary structures representative of magnetic flux tube bend are observed to bend from the coronal hole to the equator according to coronal images at solar minumum, and the closer the structure to the coronal hole boundary the more inclined (e.g. Forsyth and Marsch [60], Figure 1). In summary, this study [57,59] provides a physical explanation to the observed anti-correlation between the solar wind speed and the angular distance from the tube roots to their nearby coronal holes [55]. It should be pointed out that the empirical relation of the solar wind speed and the flux tube properties has been extensively used in space weather forcasting models of near-Earth solar wind speeds. Thus, the results presented here [57,59] are useful to improve these forcasting models on a physical basis.

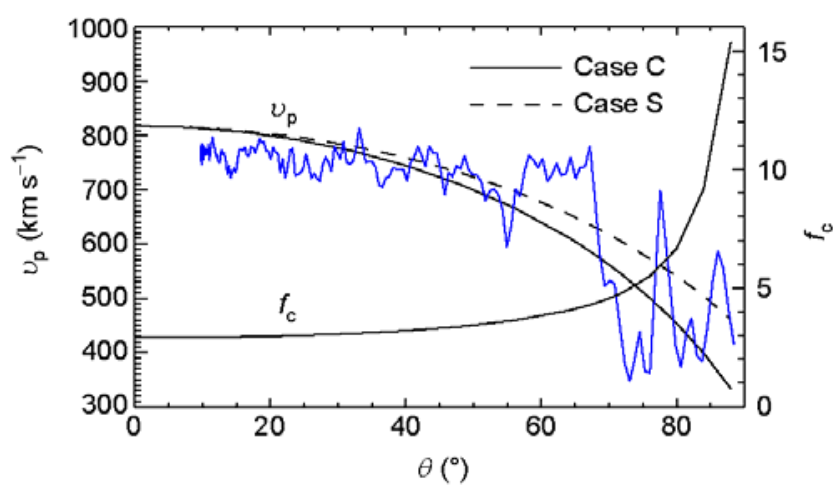

Figure 2 Variations of the solar wind speed at $1 \mathrm{AU}\left(v_{\mathrm{p}}\right)$ and the flux tube expansion factor in the corona $\left(f_{\mathrm{c}}\right)$ with the flux tube co-latitude at $1 \mathrm{AU}$ $(\theta)$. Two cases are presented with Case $\mathrm{C}$ (solid line) considering the effect of the flux tube curvature and Case $\mathrm{S}$ (dashed line) not. Blue line presents the solar wind speed measurement taken by Ulysses from 12 September 1994 to 4 March 1995 during its first polar transit (taken from [57]). 


\subsection{Persistent releases of streamer blobs and diagnostics of near-Sun slow wind speeds}

Outflowing elongated blob structures are frequently observed atop quiescent streamers [16]. They are weakly brighter than surroundings, first observed at 2-4 Rs with a length of $\sim 1$ Rs and a width of 0.1-0.3 Rs. Their size increases with distances $[10,61,62]$. Besides understanding the physics of their generations, blob studies are also important to the solar wind study. After their disconnections from the streamer tip, blobs flow passively in the solar wind. Therefore, blobs are regarded as velocity tracers of the backround solar wind. It is believed that the solar wind from the streamer region is a part of the slow solar wind [8-10]. Therefore, the speeds of blobs are representative of those of this type of the solar wind.
It is generally difficult to measure the solar wind speeds near the Sun with remote-sensing techniques. Two other methods have been applied. One method makes use of the ratio of emission intensities of spectral lines of minor ions, for example the ratio of OVI doublets $[48,63,64]$; the other method employs the interplanetary scintillation technique $[65,66]$. Both methods suffer from large uncertainties and limitations. The blob method of determining the solar wind speeds serves as an independent technique with a relatively high accuracy. Besides, both the radial and temporal evolutions of the speeds are deducible, as will be introduced later.

It is interesting to note that in some events blobs are released persistently from a streamer [61]. One such event during the days from April 19 to 26 in 1997 was reported by Wang et al. The average daily occurrence rate of blobs is
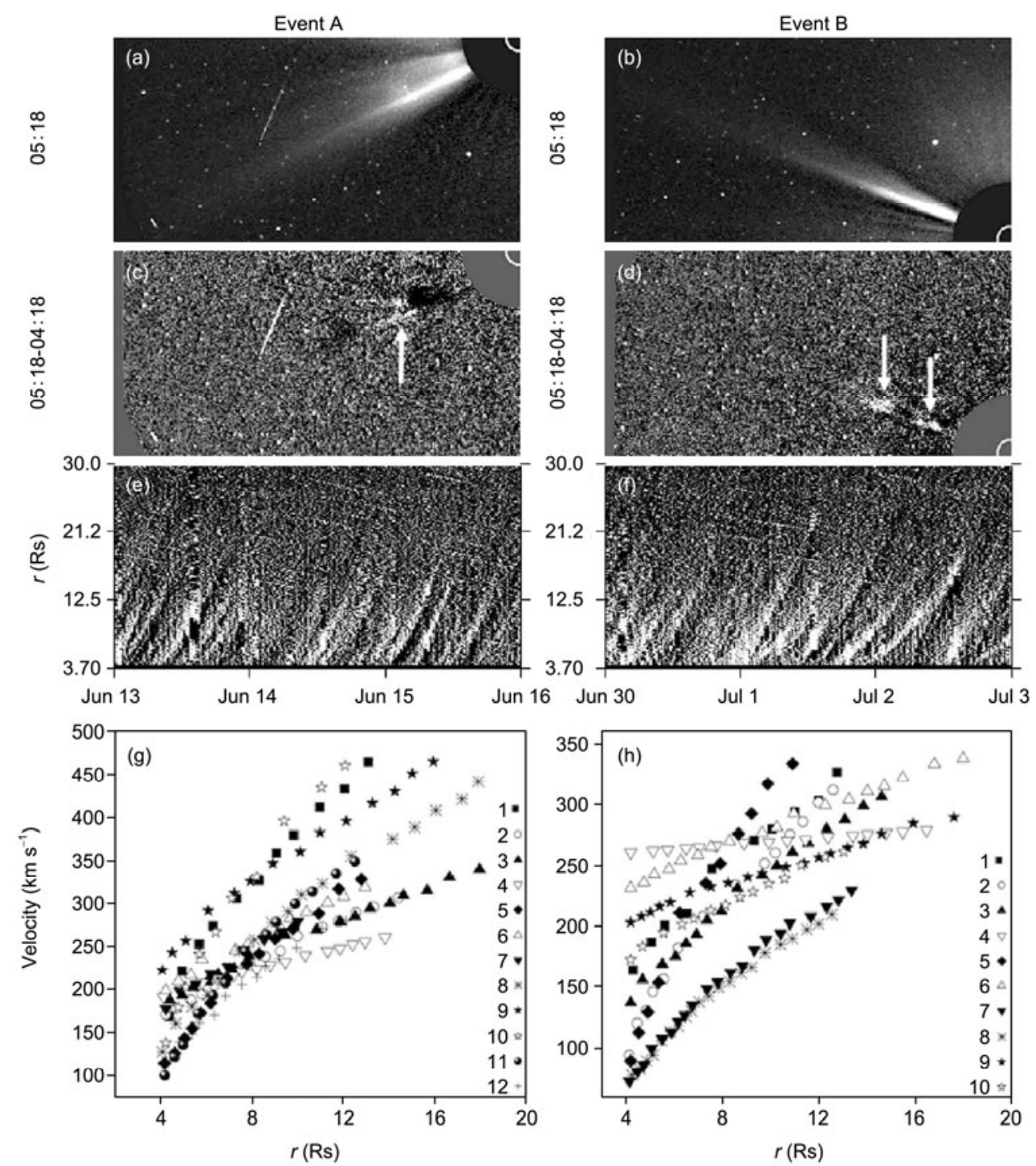

Figure 3 Two events of quasi-periodic releases of streamer blobs observed by LASCO C3, Event A (left panels: 13-15 June) and Event B (right panels: 30 June-2 July). In the eight panels, we show: background-subtracted C3 images recorded at 0518 UT on 15 June (a) and on 1 July (b), which are cropped to 30 Rs in the horizontal direction and 15 Rs in the vertical direction; the same-size difference images taken at 0518 and 0418 UT on 15 June (c) and 1 July (d); height-time tracks of blobs for Events A (e) and B (f), which are produced by stacking radial strips from successive difference images along the streamer axis; and the fitted blob velocities as a function of heliocentric distance ( $(\mathrm{g})$ and $(\mathrm{h})$ for Events A and B) (taken from [62]). 
3-5. Two similar events occurring from June 13-June 15 (Event A) and from June 30-July 2 (Event B) in 2007 observed by Song et al. [62] are shown in Figure 3. From the height-time plots of the two events (Figure 3(e) and (f)), it can be seen that 12 and 10 blobs are observed in three days. It is easy to tell the quasi-periodic behaviour of blob releases. However, this feature was hardly appreciated until Chen et al. [43] published their numerical results of blob formation.

To illuminate the physics of blob formation, Chen et al. [43] constructed an magnetohydrodynamic (MHD) model of the streamer and solar wind. It is found that the streamer develops an instability process in the cusp region, whose nonlinear development drives recurrent reconnections atop the streamer and results in quasi-periodic formations of blobs. Releases of blobs do not affect the global morphology of streamers. Thus, the streamer is stable on a large scale while unstable in the local cusp region. It is found that the instability process is closely associated with the cusp topology where the magnetic field is too weak to confine hot plasmas. Since the cusp is an intrinsic part of a typical streamer, the simulated blob formation process is dubbed the streamer intrinsic instability by Chen et al. [43]. A complete cycle of blob formation is shown in Figure 4.

The streamer instability can be divided into two stages. The first stage is characterized by the streamer elongation process, in which the cusp field lines are carried outwards by expanding plasmas due to the failure of magnetic confinement at the cusp. In this stage, streamer plasmas push into the relatively-fast solar wind region. This gives rise to a velocity shear configuration. The shear can be strong enough to trigger the so-called streaming sausage mode instability $[67,68]$, whose nonlinear development drives reconnections and blob formations. This represents the second stage. After reconnections, the streamer returns to its initial state ready to generate next blob. Besides the mentioned velocity-shear instability, other factors may also contribute to the contraction and reconnection of the elongating streamer field lines, like the pressure gradient developing after plasma expansion [10], and the converging effect of solar wind flows around the streamer [69].

According to Chen et al. [43], the period of blob generation is $4-5 \mathrm{~h}$ comparable to what observed. In early studies, only two persistent-release events of blobs were reported $[10,61]$. It is expected that more events should exist if blobs are products of streamer instabilities. Song et al. [62] conducted a survey through the LASCO data obtained in 2007 and did find 10 multi-blob events with 106 blobs in total. All events last for 3-4 d. In Figure 5 velocity profiles for all blobs observed in Song et al. [70] are plotted together. From this figure and other plots not shown here, it can be seen that all velocities increase monotonically with radial distance, and vary in a relatively small range of $<300 \mathrm{~km} \mathrm{~s}^{-1}$. These results will be compared with those of another group of blobs in the following section. Note these measurements are representative of the slow wind velocities along the plasma sheet.

Besides diagnosing the radial evolution of the slow wind velocity, one can also use these measurements to determine the temporal velocity variation at a fixed altitude by comparing the blob velocities of one event. For blobs in Event A and Event B (Figure 3), the obtained velocity variations at 6 ,

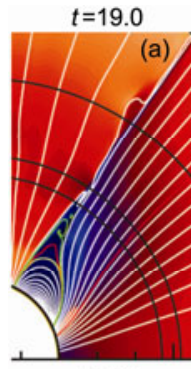

$t=21.5$
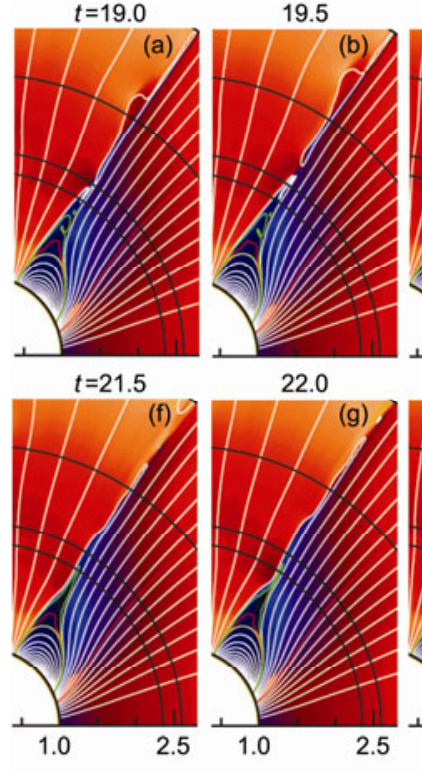

20.5

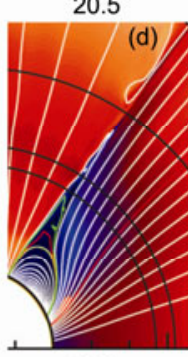

23.0

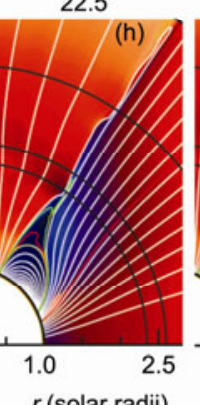

$21.0 \mathrm{~h}$

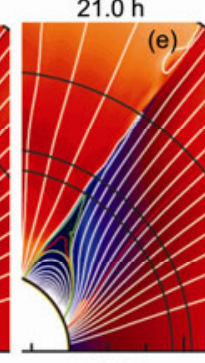

$23.5 \mathrm{~h}$

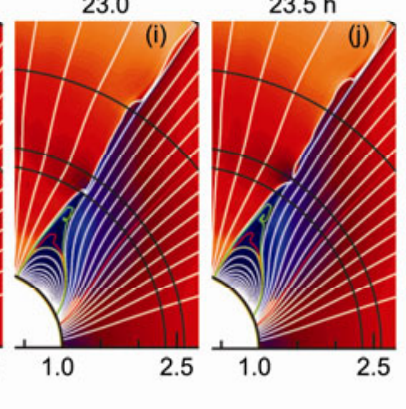

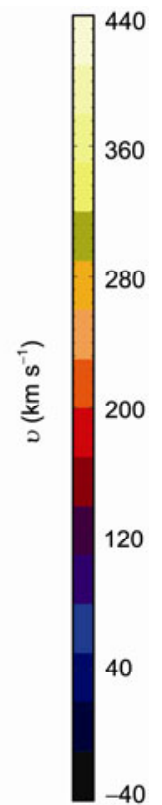

Figure 4 Ten frames, separated by $30 \mathrm{~min}$ in time, given by a $2.5 \mathrm{D}$ (axisymmetrical) MHD simulation of streamer blobs. The time spans from $t=19$ to $t=23.5 \mathrm{~h}$ increasing from left to right and top to bottom. The three black arcs are 2.35, 2.6, and 3.6 Rs away from the solar center. White lines represent magnetic field lines, the color map represents the distribution of the radial solar wind speed. The green, red, and yellow lines are shown to illustrate the blob formation process (taken from [43]). 


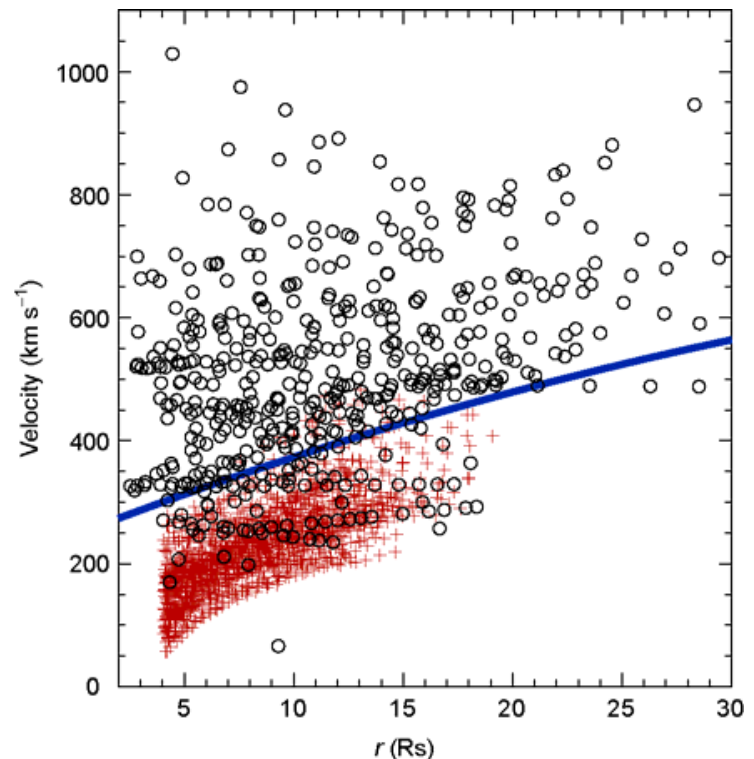

Figure 5 Velocity-distance (in units of solar radii) profiles for the 45 post-CME blobs collected by Song et al. [70] (black circles) and the 106 streamer blobs (red pluses) given by Song et al. [62]. A blue line is drawn to separate their velocity distributions, which is given by the average of the polynomial fittings to the two groups of velocity measurements and expressed as $v=-0.1 r^{2}+13.7 r+246$ with $v$ and $r$ in units of $\mathrm{km} \mathrm{s}^{-1}$ and Rs, respectively. About $83 \%$ of CME blobs have velocities above the blue line, and almost all streamer blobs ( $98 \%$ ) have velocities below this line (taken from [70]).

9 and 12 Rs are shown in Figure 6. It can be seen that the slow wind speeds can vary significantly in a range of 100 $200 \mathrm{~km} \mathrm{~s}^{-1}$ within several hours. Through in-situ measurements, it is well known that the slow wind is featured by a large variability, which may have its origin near the Sun as indicated by this study. This is the first time the observational evidence of the slow wind speed variability near the Sun is presented.

\section{Studies on Coronal Mass Ejections (CMEs)}

\subsection{Energy release mechanisms of CMEs: MHD studies with a flux-rope model}

It is generally believed that the energy to be released during solar eruptions is magnetic in nature [24,25]. It is also known that there are no violent plasma and magnetic reconfigurations prior to solar eruptions, therefore, the energy to be released has been gradually transported to and accumulated in the corona over a relatively long term. The energy is transported via footpoint motions associated with sub-photospheric convections. This means that the pre-eruption field is highly non-potential with a significant amount of free energy. Once it reaches a certain threshold, the system tends to reach a lower energy state by releasing all or part of the free energy. The released energy is converted into the plasma kinetic and thermal energies, as well as the energies of energetic particles and radiative photons [27]. Any CME models should at least describe the pre-eruption high energy state and the energy release process.

With in-situ measurements, large-scale rotations of the magnetic field can be frequently observed within an ICME (Interplanetary CME) indicating the presence of magnetic flux rope [71]. A flux rope can be simply defined as the high-energy magnetic structure consisting of twisted field lines with significant helical current components.

It is still rather controversial regarding the existence of flux ropes in the pre-eruption corona. Many authors believe that the flux rope is a necessary high-energy structure leading to CMEs. This is mainly based on the following points. (1) Rope structures are found in ICMEs according to in-situ measurements and the numerical reconstructions of interplanetary magnetic fields [72-74]. (2) Twisted rope-like structures are frequently observed by coronagraphs during the eruption; it is possible that these rope or rope-like structures are already formed prior to the eruption. (3) CMEs are
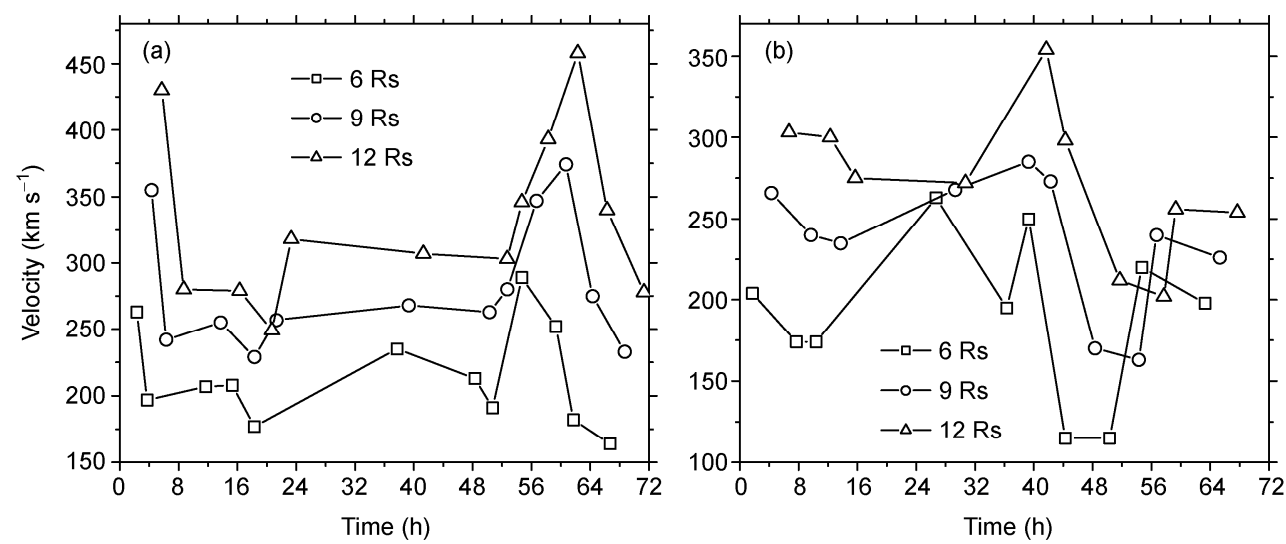

Figuer 6 Fitted velocities of successive streamer blobs at three heliocentric distances: 6 Rs (squares), 9 Rs (circles), and 12 Rs (triangles) for Events A (a) and B (b). The abscissa is the time starting from 0 UT on the first day of the event. This figure shows that the blob speed can change as much as $100-200 \mathrm{~km} \mathrm{~s}{ }^{-1}$ within a few hours at the three distances. This provides the first observational evidence of the velocity variability of the slow wind near the Sun (taken from [62]). 
closely related to filament eruptions [75], and it is generally believed that many filaments are supported by magnetic dips associated with a flux rope [76]. (4) Coronal cavities overlying a filament structure that are frequently observed to erupt as CMEs are considered to be the rope structures supporting the filaments [77]. (5) Presence of Sigmoid structures in the corona is consistent with the existence of highly-twisted magnetic fields before the eruption [78]. (6) In some events, extrapolations of coronal magnetic fields with the photospheric field measurements did exhibit the flux rope structure [79]. In latest studies with multi-wavelength high-resolution SDO data, more evidences of twisted flux rope structures prior to or during solar eruptions are reported [80,81].

Several mechanisms of flux rope formation in the corona have been proposed: (1) the structure may emerge from the sub-photosphere; (2) the rope is formed through a long-term reconnection process driven by the shear, twist, and rotation of magnetic footpoints; recent SDO studies discovered solar tornados providing a natural mechanism of rope formation [82-84]; (3) the rope is formed through fast reconnections at the early phase of the eruption. It is possible that different mechanisms may work together attributing to the formation of a flux rope. Since the flux rope configuration is a natural high-energy state which is relatively easy to simulate, it is included in most CME models.

CME models can be classified into flux-rope models and non flux-rope models according to the rope presence prior to the eruption. In the former models, the pre-exisiting flux rope carries enough free energy driving the eruption. Both analytical rope models [24,85] and numerical rope models $[29,86]$ have been developed under 2-D configuration. In these models, the rope system first evolves slowly with the boundary or the background conditions till a certain threshold is reached. The system then becomes unstable and loses equilibrium to release the free energy by throwing the rope structure into the interplanetary space. In 3-D situation, flux rope torus and kink instabilities can develop under certain conditions of the magnetic flux and twist of the rope [87-89]. On the other hand, in most if not all non flux-rope models, like the sheared-arcade [90] and break-out models [91], a rope structure is always formed rapidly through fast reconnections, which then evolves into the CME ejecta. In the following text, studies on the eruption physics will be reviewed under the framework of axisymmetric (i.e. 2.5-D) numerical flux rope models $[29,92,93]$.

It is known that a significant part of magnetic energy is converted into the flux rope kinetic energy through the work done by the Lorentz force on the rope currents. Therefore, a force analysis is necessary to understand the energy release process. Prior to the eruption, the flux rope can adjust its magnetic field-current distribution quiescently. During this phase, the magnetic field can be assumed force-free, i.e. the sum of magnetic forces vanishes. During the eruption, the force-free assumption is broken, and the sum of forces points anti-sunwards. The Lorentz forces acting on the rope currents can be divided into three groups, the rope selfforces acted by the rope magnetic fields, the forces from the background fields, and the forces from the fields associated with the current sheet that is formed during the eruption [92]. It is a simple fact that the strongest anti-sunward force is the one with the most contribution to the conversion of magnetic to flux rope kinetic energies.

For a quantitative analysis, Chen et al. [92] calculated all the above force components with the method of Green's function for an axisymmetric flux rope system. The results are shown in Figure 7. It can be seen that the most important anti-sunward force is provided by the azimuthal current inside the rope and its sub-photospheric image. This force is traditionally termed as the self force acting on the flux rope current by its own field $[94,95]$. Under equilibrium conditions, the self force is mainly balanced by the sunward force from the background field. During the eruption, both components decrease rapidly while a new restoring force provided by the newly-formed current sheet rises to be the dominant sunward force.

It can be seen that the conversion of magnetic to flux rope kinetic energy is mainly through the work done by the self force. Once the resistive MHD effect is included, reconnections set in across the CME current sheet, which greatly reduce the corresponding sunward force resulting in significant additional acceleration of the flux rope. Therefore, reconnection-induced CME accelerations are achieved mainly through the work done by the self force that is apparently-enhanced due to reconnections.

Chen et al. [92] did not consider the effect of the solar wind, neither the action of the gravity and the thermal pressure gradient force. In a following study, Chen et al. [93] improved the model by considering these factors with a polytropic process heating the corona and accelerating the solar wind. A typical streamer-solar wind configuration is obtained, within which a flux rope structure was emerged from the lower boundary. By adjusting the flux rope parameters, they were able to determine the energy threshold of the flux rope instability and simulate its catastrophic eruption. Chen et al. [93] managed to eliminate numerical reconnections across the CME current sheet and achieved numerical simulation of the flux rope eruption within the framework of ideal MHD. This is done by taking advantage of the fact that the magnetic flux function being a known constant along the current sheet [86]. This allows them to make a first-time quantitive comparison of energy releases for cases with and without magnetic reconnections. Corresponding numerical results are presented in Figure 8.

There exist two fundamentally-different release processes of magnetic energies during a flux rope eruption, including the macro-instability of the flux rope system as an ideal MHD process and magnetic reconnection across current sheets as a resistive MHD process. It has been shown by Chen et al. [93] that CMEs, even fast ones, can be simulated 

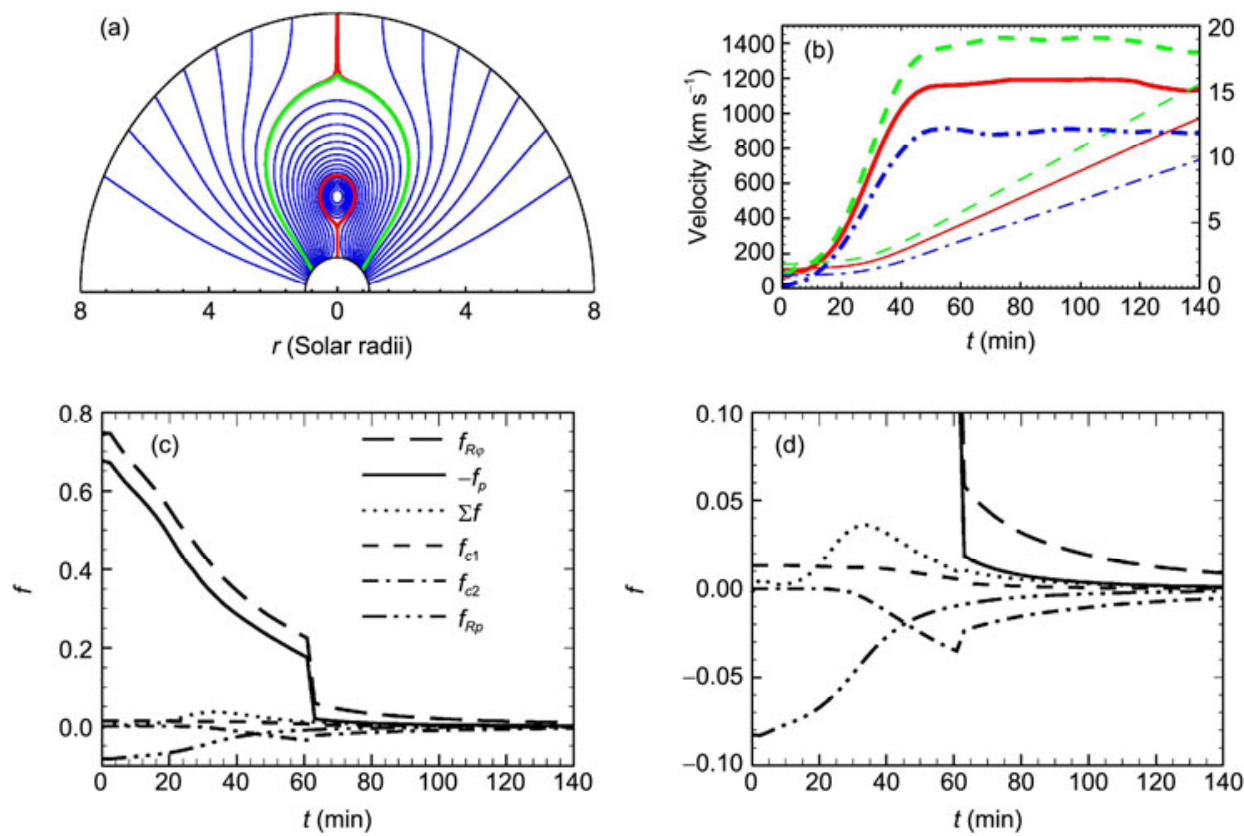

Figure 7 Flux rope eruption calculated with a 2.5 D MHD flux-rope model for CMEs. (a) Magnetic configuration of the system, the red circle and line below represent the outer border of the flux rope and the newly-formed current sheet, the green curve and the upper red line are the outermost closed field line of the streamer and the HCS; (b) temporal profiles of speeds (thick lines) and heliocentric distances (thin lines) of the top (green dashed), axis (red solid), and bottom (blue dot-dashed) of the flux rope; (c) temporal profiles of various magnetic force components acting on the flux rope currents. From top to bottom, they are the self force of the flux rope azimuthal current, the negative of the force by the background potential field, the sum of forces, the force by the HCS current, the force by the current in the newly-formed current sheet, and the self force of the rope poloidal current; (d) enlargement of the force profiles in the $y$-axis range of $[-0.1,0.1]$ (taken from [92]).

with the flux rope model even in the absence of magnetic reconnection. It is also shown that significant additional acceleration of CMEs can be achieved once reconnection sets in (Figure 8). Quantitative comparison between the two solutions is shown in Figure 8 indicating that the energy released by the ideal macro-flux rope instability and that released via reconnections are comparable. This means that both energy-release processes are important to CME dynamics.

It should be emphasized that a significant part of the energy release of reconnections is via the work done by the flux rope self force. As mentioned, the self force is apparently enhanced as the sunward force associated with the CME current sheet is weakened by reconnections. Reconnections also produce rapid exhausts. These exhausts may cause additional flux rope acceleration [28]. Nevertheless, it is believed that the former process is the main mechanism of CME accelerations induced by reconnections. More discussion along this line will be presented in the last section.

Furthermore, a smooth transition from fast to slow CMEs is observed when varying the background magnetic field strength (Figure 8). In a stronger field more free energy can be stored and released to drive a faster CME. This indicates that the two types of CMEs, the fast and slow ones, which were defined observationally [96,97], may be physically identical. This is consistent with recent statistical studies of CMEs based on a large sample of events [98-100]. Finally, the simulated eruption shown in Figure 8 is featured by the three-phase dynamical evolution including the initiation, main acceleration, and propagation phases agreeing with the observational finding of Zhang and Deer [100].

\subsection{Studies on magnetic islands of reconnections along the CME current sheet}

The current sheet structure formed behind a CME plays a crucial role in both $\mathrm{CME}$ and flare processes, since reconnections across which account for the energy release of flares, and contribute significantly to CME accelerations. One typical product of reconnections is magnetic islands or plasma blobs, which are observable with coronagraphs when they are denser than surroundings. In this sub-section, we focus on these coronal structures observed along the presumable current sheet behind a CME to infer the properties of coronal reconnections and associated electron acceleration mechanism.

(i) A statisfical study on magnetic islands and discussion on magnetic reconnections in the outer corona. Two different large-scale current sheet structures exist in the outer corona. One is the HCS on top of the streamer cusp, extending into the heliosphere and can be measured in-situ, whose formation is closely related to coronal expansion and solar wind acceleration. Some studies on the slow solar wind and plasma blobs related to HCS have been reviewed 

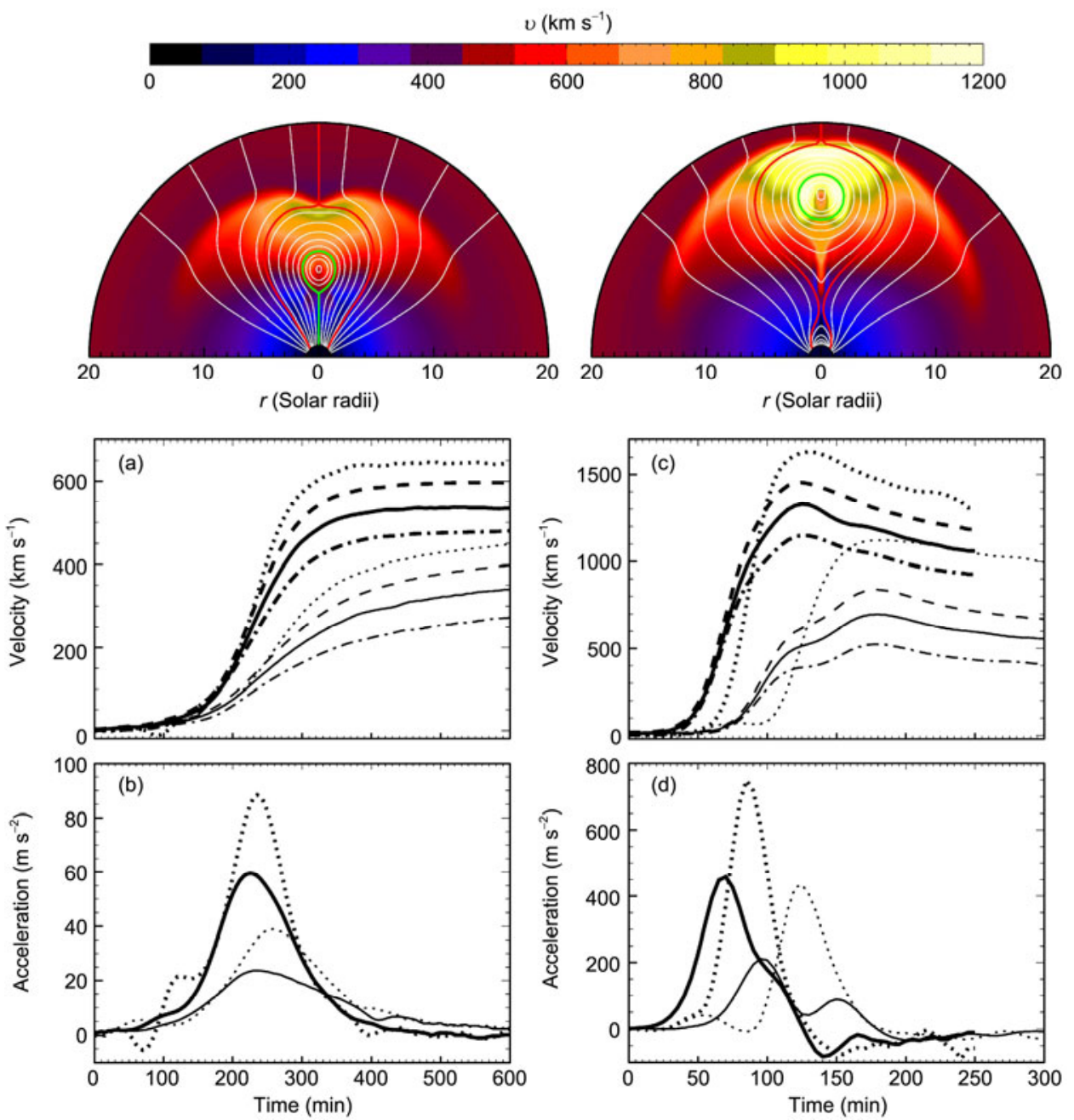

Figure 8 Upper panels: velocity contour and magnetic configuration at the same time after the start of the eruption as calculated with a flux-rope CME model considering the effect of the solar wind. The magnetic field strengths at the pole for both cases are taken to be $12 \mathrm{G}$. The left panel is for ideal MHD calculation eliminating the effect of reconnection, while the right panel is for resistive MHD calculation considering the effect of reconnection. The red circle and line below represent the outer border of the flux rope and the newly-formed current sheet, the green curve and the upper red line are the outermost closed field line of the streamer and the HCS; Middle and lower panels (a)-(d): velocity and acceleration profiles at the streamer cusp (dotted), the rope top (dashed), the rope axis (solid), and the rope bottom (dot-dashed) for cases taking the magnetic field strength at the pole to be $4 \mathrm{G}$ (left) and $20 \mathrm{G}$ (right), the thick (thin) lines are for the ideal (resistive) MHD solutions (taken from [93]).

in the above section. Here we focus on the other type of current sheet that is formed during a CME eruption.

Both the classical CSHKP flare model [101-104] and recent CME models [105] predicted the existence of a long current sheet connecting the post-flare loops and the CME ejecta. Nevertheless, the current sheet, as a magnetic structure, is not directly observable at this time. Its existence and physical properties are inferred through indirect observations. For instance, a bright ray is frequently observed in the wake of CMEs [70,106-111], which is presumed to contain a current-sheet structure. This presumption is supported by the following observations. First, in many events the ray links the top of the post-flare loops to the bottom of the CME ejecta (see Figure 9 for a typical event), consistent with theoretical expectations as mentioned. Second, enhanced high-temperature emission from ions like FeXVIII (with a formation temperature of $\sim 6 \mathrm{MK}$ ) is observed along CME rays according to UVCS data [112], this indicates that the plasma there is much hotter than surroundings [106] agreeing with the idea that plasmas are heated by reconnections along the current sheet. Third, plasma blobs are sometimes observed to move outwards along the ray, which are believed to be products of reconnections along the current sheet.

It has been suggested that the ray as a whole represents a significantly broadened current-sheet structure $[108,109]$. The ray is as wide as tens to hundreds of kilometers, wider by several orders of magnitude than that predicted by classical plasma theory [113,114]. Broadening mechanisms related to turbulent reconnections associated with tearing mode instabilities or time-dependent Petschek reconnections have been proposed [115]. 


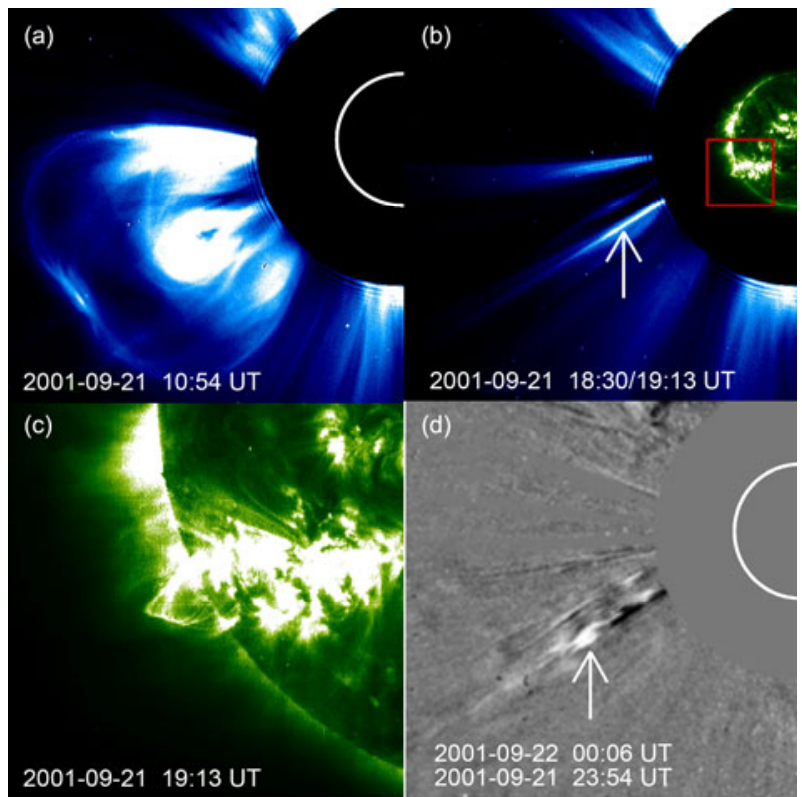

Figure 9 A typical event with post-CME ray, blobs, and loops. (a) The white-light CME image observed by LASCO C2; (b) composite image from C2 (18:30 UT) and EIT $195 \AA$ (19:13 UT) showing the post-CME ray, as denoted by the white arrow, and the post-CME loops; (c) the enlarged EIT image inside the red square in (b); (d) running difference image showing the presence of a blob structure as indicated by the arrow (taken from [70]).

It is also argued that the post-CME ray corresponds to a plasma sheet with an embedding current sheet structure, similar to the HCS-plasma sheet configuration atop a streamer [116]. In-situ measurements show that the HCS is much narrower than the associated plasma sheet [117]. Therefore, the requirement on the broadening mechanism can be considerably reduced. Another scenario has been proposed by Vrsnak et al. [111]. They suggested that the ray is given by the exhaust of a Petschek reconnection. Yet, the corresponding diffusion region is too low and thin to be observable with present coronagraphs [118]. According to this scenario, the ray structure is constrained by a pair of slow mode shocks that heat and accelerate relevant coronal plasmas, containing no current sheet structure. It is not possible yet to tell which scenario is valid based on present observations.

Plasma blobs are important signatures of reconnections which are observable with coronagraphs. So far, only a few CME-ray-blob events have been studied in detail [108,109]. According to these studies, the initial blob velocities are close to the reconnection outflow speed, or approximately the local Alfvén speed. Given the plasma densities, the magnetic field strength along the current sheet can be deduced. For example, for the 8 January 2002 event the field strength along the ray is estimated to be 0.69 gauss $(\mathrm{G})$ at 3.1 Rs and $0.47 \mathrm{G}$ at $4.5 \mathrm{Rs}$ by Ko et al. [108]. In Lin et al. [109], the reconnection rate is estimated for the 18 November 2003 event with the inflow speed determined with UVCS.
Recently, Song et al. [70] found 11 well-defined CMEray-blob events observed by LASCO in SC 23. 45 blobs are observed in these events, whose velocities are shown in Figure 5 together with those of the streamer blobs. The blue line in the figure is given by $v=-0.1 r^{2}+13.7 r+246.9$, where $v$ and $r$ are in units of $\mathrm{km} \mathrm{s}^{-1}$ and Rs. It is obvious that the line serves as a good separator of the speed distributions of the two groups of blobs. It is found that $\sim 83 \%$ of the CMEblob velocities are above the line, while almost all (98\%) of the streamer-blob velocities below. It is also found that CME blobs and streamer blobs have different velocity variations with distances. Most if not all streamer blobs present a gradual monotonic acceleration, while of CME blobs $58 \%$ (20\%) being accelerated (decelerated) and 22\% moving with nearly constant speeds. And CME blobs are generally faster than streamer blobs. At a fixed distance, the speeds of CME blobs vary in a range of $400-800 \mathrm{~km} \mathrm{~s}^{-1}$ much wider than that of streamer blobs $\left(\leqslant 300 \mathrm{~km} \mathrm{~s}^{-1}\right)$.

It is suggested that the two types of blobs are formed from physically-different reconnection processes based on the following arguements. Firstly, the blobs, as reconnection products, show different dynamical behaviors. Secondly, the reconnection processes are associated with different magnetic structures being either the post-CME current sheet or a streamer-cusp region with different strengths of magnetic field. Last, their triggering processes may be different with the post-CME reconnection being driven mostly by tearing mode instabilities $[108,109]$ and the streamer-blob reconnection being driven possibly by fluid instabilities associated with the failure of plasma confinement at the cusp [43].

(ii) First observation of magnetic island coalescence and associated electron acceleration. A significant part of the magnetic energy is used to accelerate electrons during reconnections. Different mechanisms of electron acceleration by reconnections have been summarized in Oka et al. [119]. Two important ones are called as the $\mathrm{X}$ type and $\mathrm{O}$ type. The former process corresponds to electron acceleration by induced electric field at the $\mathrm{X}$-like reconnection region, and the later process refers to the electron acceleration by the rapid contraction of the O-like magnetic islands [120,121]. Recent numerical studies indicate that the coalescence of two approaching magnetic islands (also called anti-reconnection) is very efficient in electron acceleration [119].

Electron energization at magnetid islands has been reported with the Cluster observations during reconnections in the Earth's magnetosphere [122-124]. This provides support to the above scenarios. However, evidence of electron acceleration by magnetic island coalescence has not been found until Song et al. [37] published a study on a CME observed by STEREO [125]. They reported the first observation of colascence of magnetic islands (i.e. plasma blobs) near the Sun and relevant radio signals produced by energetic electrons, as will be introduced in the following text.

According to the data from STEREO coronagraphs 
COR1/2 on 23 May 2010, a long and bright ray structure is present behind the CME, along which two outward-moving blobs are detected (Figure 10). The blobs enter into the COR1 FOV at 04:54 UT and 06:39 UT on May 24, respectively. Their average speeds are 187 and $361 \mathrm{~km} \mathrm{~s}^{-1}$. At 08:09 UT, the two blobs meet and start to merge. The merging lasts for about $2 \mathrm{~h}$. The merged-together blob moves at a speed of $\sim 326 \mathrm{~km} \mathrm{~s}^{-1}$. In the meantime the blob shape changes significantly from a long-thin structure into a more circular one.

Between 10:09-10:24, there appears an opening-up feature on top of the blob as seen from the image sequence shown in the left-bottom panels of Figure 10.

To tell whether energetic electrons are produced, Song et al. [37] examined the radio data of WAVES/STEREO [126] and found a fast-drifting type-III like radio burst at $\sim 10: 23$ UT (Figure 11) starting at $\sim 3.7 \mathrm{MHz}$. This type of radio burst is caused by energetic electrons propagating antisunwards. To determine the physical connection between the burst and the blob dynamics, Song et al. estimated the emission distances of the radio signals with two different density distributions, the Newkirk density model [127] and the online real-time modeling result provided by the Predictive Science STEREO modeling group (http://www.predsci. com/stereo). With either model the emission source is estimated to be at $\sim 8$ Rs. From the COR 2 data the opening up is seen to take place at a similar distance, and temporally coincident with the radio burst. This indicates that they are physically related. The opening up is interpreted to be caused by reconnections as the blob after coalescence gets faster to catch up overlying field lines. The electrons are accelerated by the coalescing process and then confined in the closed magnetic structure till the opening up releases them into the open field reigon. This gives rise to the bumpon-a-tail velocity distribution of electrons, a necessary condition for exciting Langmuir waves and radio bursts. Besides, time-scale estimates of electron acceleration based on the equations given by Drake et al. [120] for contraction and by Oka et al. [119] for coalescence of reconnecting magnetic islands also support the scenario that electrons are accelerated mainly by the coalescing process.

In addition, Song et al. [37] reported that before the coalescence the first blob shows a two-core peanut-like shape as pointed out with yellow arrows in Figure 10. This was suggested to be a result of reconnection at the middle part of the magnetic island during its elongation. Thus, three different reconnection processes are observed in this event, as illustrated in the schematic diagram of Figure 10. Using the axisymmetric flux rope model of CMEs [93], Song et al. [37] simulated the eruption process. The results are shown in Figure 12. The modeled CME velocities and formation and

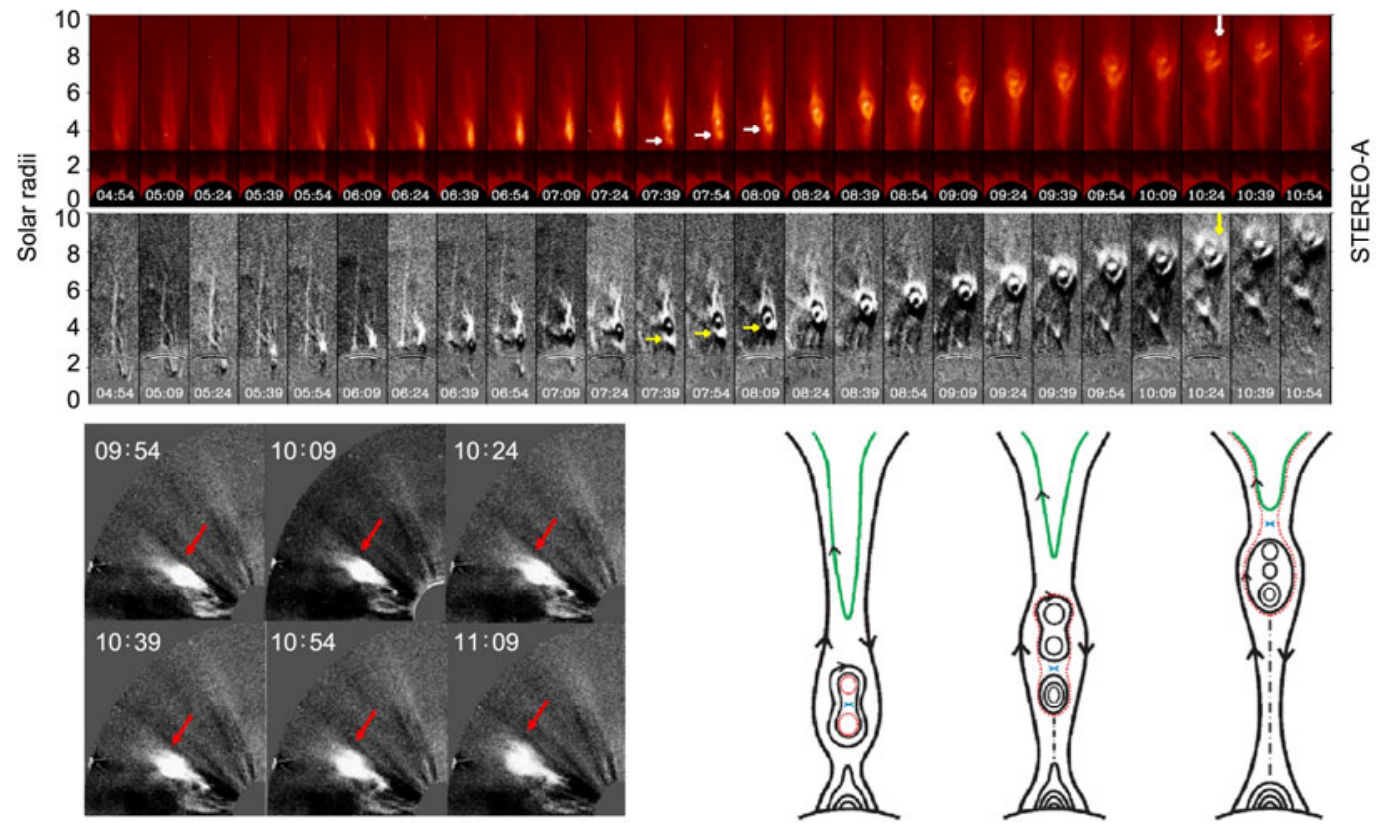

Figure 10 Upper two panels: white-light and running-difference images of magnetic blobs along a post-CME ray observed by the STEREO-A COR1/2 coronagraphs. The first blob evolved into a peanut shape in the 07:39 frame. White arrows indicate the second blob moving and catching up the first blob. Between 10:09 and 10:24, the upper right part of the blob appeared to open up (as indicated by the arrows), suggesting possible reconnection with the open magnetic-field lines lying above. Lower left panel: The base-difference images showing the blob opening-up. The base image for these plots is from the frame with time 04:24. The opening-up phase is clearly seen from the 10:24 frame (and thereafter), although it is possible that it may have started in the 10:09 frame. Lower right panel: Drawings show the three reconnection processes in this event. Left: The breaking of the first blob into a two-core-peanut blob. Center: The merging of the two blobs. Right: The reconnection between the merged blob with the overlying magnetic field. Blue crosses mark the reconnection sites. Field lines before the reconnection are shown as black and green solid lines; field lines after the reconnection are shown as red dotted lines (taken from [37]). 


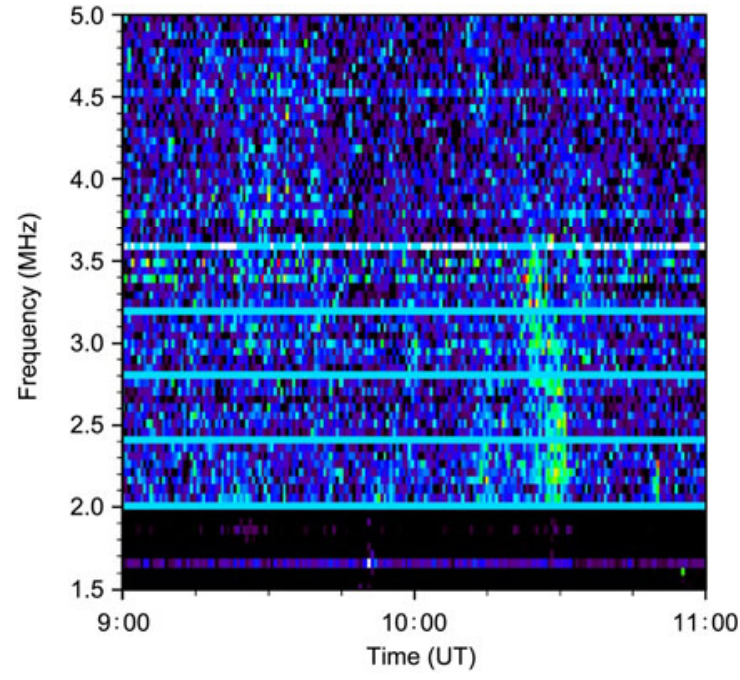

Figure 11 Type-III like radio burst observed by WAVES on board the STEREO-A spacecraft. The burst occurred around 10:23 UT starting from a frequency of $3.7 \mathrm{MHz}$ (taken from [37]).

coalescence of blobs along the current sheet behind the CME agree well with observations.
It should be emphasized that this work presents the firsttime observation of coalescing magnetic islands with relevant signatures of energetic electrons in space. It provides an important clue to understanding how electrons are accelerated during reconnections in a more general sense.

\section{White-light and radio manifestations of CME-streamer interaction}

As the large-scale eruption, CMEs cause strong disturbances to nearby coronal structures. Specifically, CMEs frequently interact with streamers. It is well known that many CMEs originate from active regions beneath a streamer, therefore CMEs are frequently associated with overall or partial distruption of streamers (e.g. blowout and puff CMEs: $[20,22])$. Besides, CMEs may also erupt from a source not apparently associated with a streamer. In these events, the streamer is affected by the lateral expansion of the ejecta and associated disturbances.

For example, the streamer stalk may present a strong deflection along with an outward propagating kink upon the
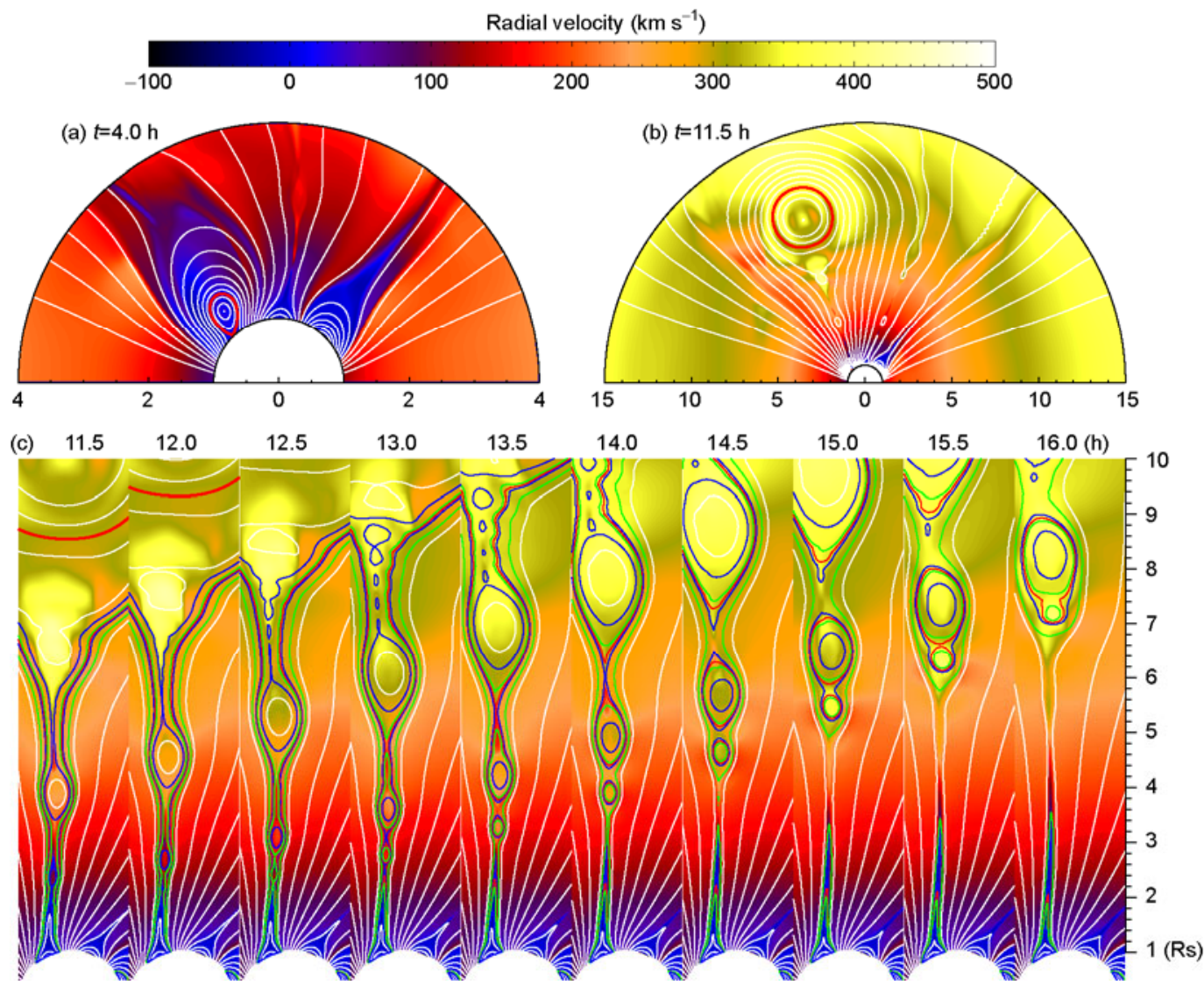

Figure 12 Numerical simulations of the generation and merging of multiple blobs in the current sheet behind a flux rope eruption with a 2.5-D axisymmetric flux-rope CME model. (a), (b) Snapshots at times $t=4.0 \mathrm{~h}$ and $t=11.5 \mathrm{~h}$, respectively. White lines are magnetic fields. The colors depict the radial velocity, matching the legend across the top of the figure. The flux rope is represented by the red circles in (a), (b), that emerges from the bottom center of the leftmost streamer. (c) is a time sequence of 10 frames, $30 \mathrm{~min}$ apart, from $t=11.5 \mathrm{~h}$ to $t=16.0 \mathrm{~h}$. It represents a plot enlargement along the current sheet behind the CME. The width of the slide (i.e. the horizontal size) in each frame in (c) is 2 Rs. The modeling results of the CME and blob motions are in general consistent with what observed (taken from [37]). 
impact of CMEs. This phenomenon has been used to trace the CME-driven shock [36]. In addition, reconnections across the streamer current sheet may have been triggered during a CME eruption, as indicated by the observed streamer disconnection [17,128,129], release of plasma blobs [37], and streamer in/out pairs [17].

Data analyses combining simultaneous radio and white light observations reveal that CME-streamer interactions are important sources of type II radio bursts [130]. For example, Reiner et al. [131] suggested that the type II emission of their study is originated from enhanced density regions of the corona, and the data are consistent with an origin in the dense streamers. Cho et al. [132] calculated the type II shock heights along different position angles (PAs) based on the 2-D density distribution given by the MK4 $\mathrm{pB}$ data, which were compared with the CME-front heights along different PAs observed by MK4. They concluded that the type II burst was generated at the interface of the CME flank and the streamer. In a follow-up study, Cho et al. [133] examined $19 \mathrm{CME}$ events observed by MK4 with type IIs and found nearly half of them are associated with CMEstreamer interactions.

Other relevant studies discussed the origins of multi-band type IIs with a few separate type II episodes with different starting time, frequencies, and drifts observed within a period of several to tens of minutes [134]. It has been suggested that these events are either from different locations along a single coronal shock [134] or from shocks of different origins [135]. Cho et al. [136] analyzed one such event dated on 31 December 2007, and concluded that one band is from the CME-streamer interaction region. In addition, Mancuso and Abbo [137] reported the bifurcation of a metric type II burst, in which the radio emission drifts to both higher and lower frequencies, as a result of the bi-directional shock propagation along the streamer stalk.

In recent studies, we have discovered several interesting white-light and radio phenomena associated with CMEstreamer interactions, including streamer waves manifested as the wavy motion of a streamer stalk [138-140], and spectral bump and break features of type II radio busts caused by the radio source propagating through a streamer from outside [141] and inside of the streamer [142]. These studies will be reviewed as follows.

\subsection{White-light observations: Streamer waves and associated coronal seismological studies}

Considering the fundamental role played by waves in a disturbed magnetized plasma system, one may ask a natural question: can the streamer, as the most prominent largescale structure in the corona, respond in the form of observable waves or wavelike motions to a strong impact from a CME? A well-developed streamer is featured by closed field arcades and a long thin plasma sheet rooted on the photosphere. The plasma sheet extends from the streamer cusp to the deep heliosphere like a bright belt flying in the solar wind with one end tied to the solar surface. Streamer deflections and kinks have been observed upon the CME impacts, however, no one considers the possibility of CMEinduced streamer wavy motions.

Intuitively, a magnetic restoring force pointing to the original equilibrium position arises once the streamer is deflected. Given a fast-moving and expanding eruption and a large streamer deflection, the restoring force may be strong enough not only to deflect but also oscillate the streamer structure. This intuitive picture has been verified by Chen et al. (2010) [138], as will be described in the following.

(i) Observational features and formation meshanism of streamer waves. Streamer waves are first reported by Chen et al. [138] with the LASCO data. The wave properties including the wavelength, wave period, and phase speed are deduced. In a follow-up study, Chen et al. [139] develops a novel seismological method based on these measurements to assess the radial and temporal evolutions of the coronal Alfvén speed and magnetic field strength along the plasma sheet. Both studies focus on the 6 July 2004 event. In a third paper, Feng et al. [140] present 8 candidate events observed during SC 23 (Table 1).

With this table and relevant LASCO movies, one can summarize several common features of the listed events: (1) the driving CMEs are wide and fast with a linear speed $>$ $\sim 1000 \mathrm{~km} \mathrm{~s}^{-1}$, an average speed of $1530 \mathrm{~km} \mathrm{~s}^{-1}$, and 6 being halo events; (2) all front-side CMEs have accompanying flares indicating occurrence of fast reconnections; (3) in most events the bright ejecta hits the streamer directly, and the interaction starts at a distance below the C2 FOV $(\leqslant 2.2$ Rs). These features shed light on the excitation conditions of streamer waves, as will be discussed later.

To illustrate the formation and propagation of a streamer wave, in Figure 13 we present the 6 July 2004 event. The CME linear speed is $1307 \mathrm{~km} \mathrm{~s}^{-1}$, the interaction is observed since 20:30 UT. At the bottom of C2 FOV the streamer is strongly deflected indicating the interaction starts even lower. At 20:58 UT, the CME left the FOV, while the streamer starts to bounce back and overshoots. This gives rise to the streamer oscillation, which propagates outwards along the plasma sheet. Accordingly, there appear "black-white and white-black" pairs on the difference images, which have been used by Chen et al. [138] and Feng et al. [140] to delineate the wave profiles. They further determine the locations of observable wave troughs and crests at all exposure times to calculate the wave paramteres. Based on this case study, Chen et al. [138] present the observational definition of streamer wave as a long-period largescale wave phenomenon excited in the aftermath of CMEstreamer interaction, which is carried by and propagating outwards along the plasma sheet, representing one of the largest wave phenomena ever observed in the corona.

As mentioned, the deflected streamer gives rise to a magnetic restoring force due to the photospheric line-tying 
Table 1 Relevant parameters of CMEs and streamers for the eight candidate streamer wave events observed during SC $23^{\text {a) }}$

\begin{tabular}{|c|c|c|c|c|c|c|}
\hline \multicolumn{6}{|c|}{ CME } & \multirow{2}{*}{ Streamer CPA $\left(^{\circ}\right)$} \\
\hline Date & Time (UT) & $\mathrm{CPA}\left({ }^{\circ}\right)$ & Width $\left(^{\circ}\right)$ & Speed $\left(\mathrm{km} \mathrm{s}^{-1}\right)$ & Flares & \\
\hline 2001-04-20 & $10: 06: 05$ & 65 & 127 & 1160 & - & 15 \\
\hline $\begin{array}{l}2003-05-27 * \\
(2003-05-28)\end{array}$ & $\begin{array}{c}23: 50: 05 \\
(00: 50: 05)\end{array}$ & $\begin{array}{l}\text { Halo/S } \\
\text { (Halo/BA) }\end{array}$ & $\begin{array}{c}360 \\
(360)\end{array}$ & $\begin{array}{c}964 \\
(1366)\end{array}$ & $\begin{array}{c}X 1.3 \\
\text { (X3.6) }\end{array}$ & $\begin{array}{c}185 \\
(185)\end{array}$ \\
\hline 2003-06-05 & $20: 06: 05$ & 230 & 239 & 1458 & - & 316 \\
\hline 2003-11-18* & $08: 50: 05$ & Halo/BA & 360 & 1660 & M3.9 & 319 \\
\hline 2004-07-05* & $23: 06: 05$ & Halo/BA & 360 & 1444 & - & \multirow{2}{*}{225} \\
\hline 2004-07-06 & $20: 06: 06$ & Halo/BA & 360 & 1307 & - & \\
\hline 2005-01-15 & $23: 06: 50$ & Halo & 360 & 2861 & $\mathrm{X} 2.6$ & 240 \\
\hline 2006-11-06 & $17: 54: 04$ & 80 & 80 & 1994 & $\mathrm{C} 8.8$ & 128 \\
\hline
\end{tabular}

a) The first to sixth columns present the appearance date and time (UT) of the CME ejecta in the C2 FOV, the CME type or the central position angle (CPA), angular width, the linear speed, and the GOES X-ray flare levels. The last column gives the CPA, measured at 5 Rs, of the streamer in study. Most data are from the CDAW datacenter. The events with * represent questionable wave events, in which the streamer wavy motions may get disturbed by CME trailing structures (taken from [140]).

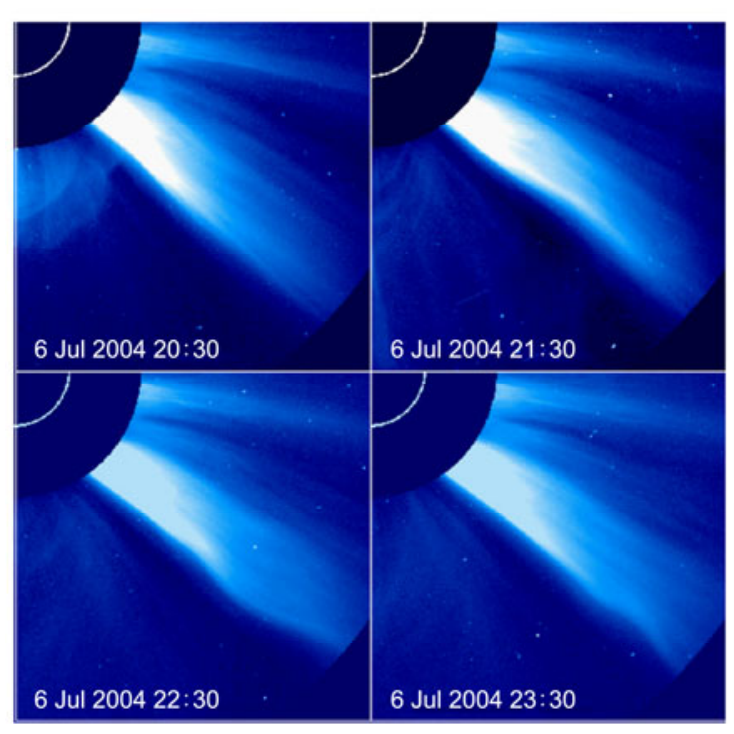

(a)
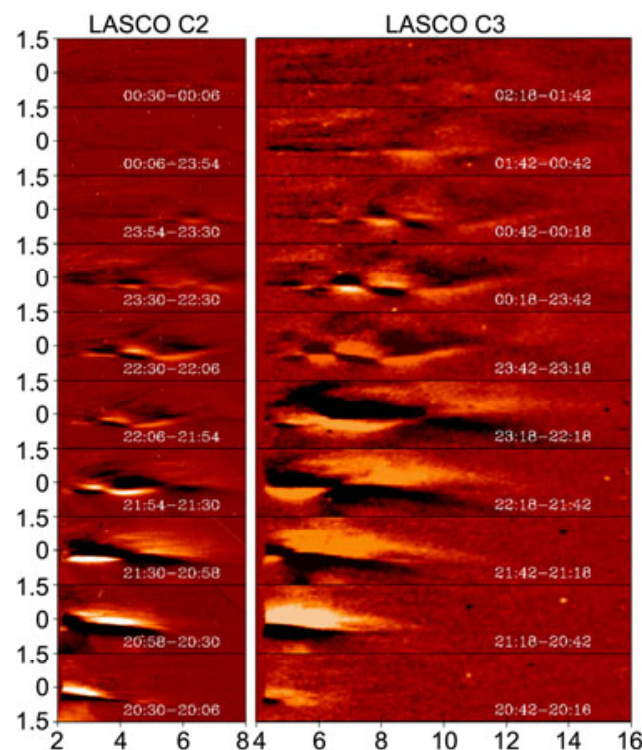

(b)

Figure 13 (a) A CME-streamer wave event observed by LASCO C2 on July 6, 2004. Only the southwest quadrants of the C2 FOV are shown. (b) Two stacks of strips cropped from C2 (left) and C3 (right) difference images. The difference times are given. Both the $x$ - and $y$-axises are in units of solar radii. The prominent white-black pairs are representative of the wavy motion of the streamer stalk (taken from [138]).

effect of its field lines. The energy received from the CME is transported outwards along with the propagation of the streamer wave, therefore, the wave amplitude becomes smaller with time. Only one to two periods of the wavy motion are recognizable with LASCO. The wave process is illustrated in the cartoon of Figure 14. Chen et al. [138] compared the wave phenomenon to a traditional Chinese dance named the "Colored Belt Dance" that is performed by dancers holding one end of a long belt in color.

Now we show how the common features of the events listed in Table 1 contribute to wave excitations. It is known that to produce a strong restoring force, the deflection should be large, and the initial interaction height should be low to deflect the closed part of the streamer. Also, both the driving CME should move and expand fast. These requirements seem to be generally satisfied by observations. The fast eruption of the CME also leaves sufficient time for the restoring force to oscillate the streamer structure. In addition, to make space for the streamer bouncing, magnetic field lines that are stretched outwards by the ejecta should close back quickly. This requires the occurrence of fast reconnections, consistent with the presence of accompanying flares with all front-side CMEs. It can be seen that all the common features summarized above favor the excitation of streamer waves.

(ii) Physical nature of streamer waves and associated seismological study. The plasma sheet, along which the streamer wave propagates, is embeded in outflowing plasmas 
(a)

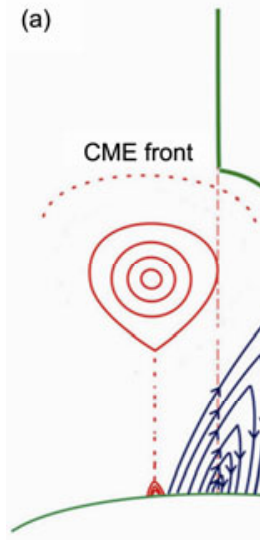

(b)

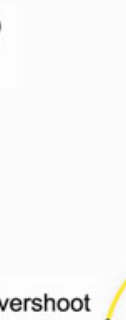

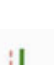

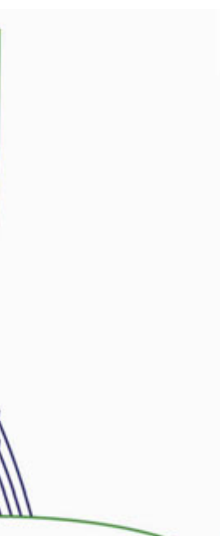

(c)

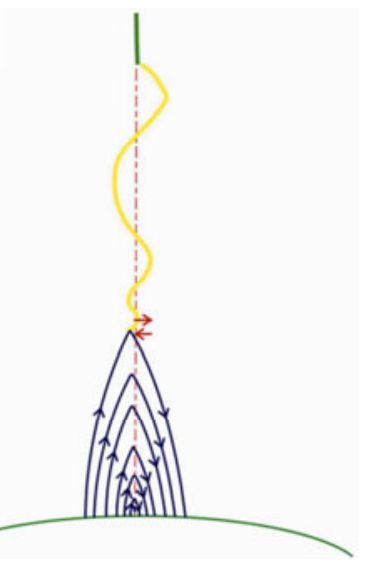

Figure 14 A cartoon illustration of streamer wave excitation in the aftermath of CME-streamer interaction. The red loops represent the CME magnetic fields, the upper dotted curve is the CME front, the lower dotted line is the current sheet in the wake of CME, the central dashed red line gives the original center of the streamer, and the yellow thick line delineates the profile of the streamer wave.

forming a sandwitch-like slab configuration. The linear wave modes supported by a plasma slab have been revealed by Edwin and Roberts [143] under reasonable simplifications. The modes can be classified into types of fast and slow, kink and sausage, body and surface. Their combinations give rise to various wave modes supported by the slab. The streamer wave belongs to the fast kink body mode according to its observational manifestation.

From the dispersion relation derived by Edwin and Roberts [143], the wave phase speed in the plasma rest frame is determined by the sonic speeds and the Alfvén speeds both inside and outside of the slab. Since the streamer wave propagates in the solar wind, the measured phase speed is given by the sum of the wind speed and the phase speed in the frame moving with the solar wind. The former can be constrained by measurements of plasma blobs $[10,16,43,62]$. The later is constrained with a parameter study on the wave dispersion relation using prescribed parameters [139], which concludes that the mode phase speed can be approximated by the exterior Alfvén speed $\left(V_{\mathrm{Ae}}\right)$ times a factor $<\sim 1$ for appropriate wave numbers and slab width. This factor is related to the dispersion effect of the density difference and velocity shear associated with the slab. Thus, the Alfvén speed along the plasma sheet can be deduced with the streamer wave measurements. With this speed and the electron number density deduced by inversion of the $\mathrm{pB}$ data, one can finally determine the magnetic field strength in the region surrounding the plasma sheet $\left(B_{\mathrm{e}}\right)$.

It should be pointed out that many coronal parameters, including the magnetic field strength, are deduced via indirect methods at this time. Similar to what done in geoseismology, coronal seismology deduces coronal parameters with wave measurements in the corona, to which the streamer wave study serves as a nice addition.

Besides the above event, Feng et al. [140] also conducted a similar seismological study on the 5 June 2003 event. From the obtained radial variations of $V_{\mathrm{Ae}}$ and $B_{\mathrm{e}}$ for both events, it can be seen that both parameters decrease monotonically with distances, $V_{\mathrm{Ae}}$ from $400-450 \mathrm{~km} \mathrm{~s}^{-1}$ at $3 \mathrm{Rs}$ to $\sim 200 \mathrm{~km} \mathrm{~s}^{-1}$ at $10 \mathrm{Rs}$, and $\mathrm{B}_{\mathrm{e}}$ from $0.1-0.15 \mathrm{G}$ at $3 \mathrm{Rs}$ to $0.01-0.02 \mathrm{G}$ at $10 \mathrm{Rs}$. Note that $\mathrm{B}_{\mathrm{e}}$ variation basically agrees with the $r^{-2}$ dependence. In addition, both parameters have a general declining trend with time at a fixed distance, possibly due to the recovering process of the post-eruption corona. Both parameters decline by about $15 \%$ during 60-90 min, i.e. in about one wave period. Of course, the coronal field does not decrease unlimitedly. Yet, it is not possible to determine how the magnetic field strength evolves after the streamer wave propagates out. Diagnostic results of the field strengths with other methods are in general consistent with the streamer wave results.

Finally, it is found that the Alfvén critical point in the slow wind surrounding the plasma sheet is at $\sim 10$ Rs where the flow speed, which equals the Alfvén speed, is $~ 200$ $\mathrm{km} \mathrm{s}^{-1}$. This agrees with the early deduction using the Helios data [144].

\subsection{Radio signatures of CME-streamer interactions}

A shock is generated as the CME is faster than the fast mode wave in the solar wind. Energetic electrons accelerated at shocks may excite Langmuir waves and type II radio bursts [130]. In the radio dynamic spectra, type IIs are narrow stripes drifting gradually from higher to lower frequencies observed in the metric to kilometric wavelengths. According to the plasma emission theory [145], their frequencies are close to the local plasma frequency and/or its harmonic. Thus, their frequency drift rate depends on the shock speed and the density gradient along the shock path.

There exist extensive studies on type II bursts [146,147]. Nevertheless, their generation mechanism and source properties remain controversial due to the lack of simultaneous white light/EUV imagings of CME shocks and high-resolution radio imagings of the bursts. For example, for metric 
type II bursts it is not sure yet whether they are driven by blast waves of flares or by a CME-driven shock, neither is it known of the exact source location of type IIs [148].

Feng et al. [141] and Kong et al. [142] proposed a promising approach to resolve the above issue by establishing physical connection between the spectral shape from the radio data and specific eruptive process observed with solar imagings. As mentioned, it is generally believed that the type II frequency is mainly determined by the electron density at the shock, the spectral shape is therefore expected to be affected by coronal structures along the shock path. Streamers are the most prominent coronal structure, several times denser than their surroundings $[9,13,15,48,149]$, it is therefore expected that the transit of a type-II shock across a streamer should result in an apparent change of the spectra. Once this causal relationship is established, it is possible to pinpoint the type II source site.

Intuitively, if the type II source (presumable a part of the shock) enters the dense streamer from a less dense coronal material outside of the streamer, the corresponding spectral band may present a temporary rise due to enhanced densities during the transit, this results in a spectral bump feature; in another case, if the type II source crosses the streamer boundary from inside, at which densities decline sharply, the spectrum may show a sudden drop of frequencies. Type II spectra with these features have been reported [141,142] and will be reviewed in the following. We also present how these studies contribute to type II source diagnostics.

Before further discussion, it is useful to present how to establish the mentioned causal relationship. This can be done through the following procedures: (1) examine care- fully the eruption to search for shock-related features like the diffuse sheath structure [34], deflections or kinks of streamer stalks and coronal rays [36], the shock distances should be measured if possible; (2) fit the type II spectrum with an appropriate density model, and convert it into the temporal variation of shock distances, which should be compared with the measured CME-shock heights for a consistency check; (3) use the spectral feature in study to infer some details of the accounting density structure, and compare with imaging observations of the structure. These procedures are followed by both studies presented below.

(i) A type II event with spectral bump. A typical type II event with a clear spectral bump feature observed on 1 November 2003 was reported by Feng et al. [141]. The dynamic spectrum is shown in Figure 15. The bump is present in the period from $22: 45-22: 55$ on both the fundamental $(F)$ and harmonic $(\mathrm{H})$ branches. The $\mathrm{F}$ branch as a whole lasts for $\sim 28 \min (22: 34-23: 02 \mathrm{UT})$ and extends from $\sim 140$ to $10 \mathrm{MHz}$ with an average drift rate of $\sim 0.08 \mathrm{MHz} \mathrm{s}^{-1}$. At 22:44 UT, both branches get intermittent, till 22:48 UT the emission becomes continuous again with a clear band splitting $[150,151]$ on the $\mathrm{H}$ branch. In the meantime, the $\mathrm{H}$-branch slope changes drastically at $\sim 40 \mathrm{MHz}$ with the average drift rate decreasing from -0.4 (22:34-22:44) to $\sim-0.04 \mathrm{MHz} \mathrm{s}^{-1}(22: 44-22: 52)$. After this, the drift rate increases slightly to $\sim 0.06 \mathrm{MHz} \mathrm{s}^{-1}$. The two solid-dashed lines in the figure are fittings to the $\mathrm{F}$ and $\mathrm{H}$ branches before 22:44 using the Saito density model [152] and a shock speed of $900 \mathrm{~km} \mathrm{~s}^{-1}$. The bump is apparently above the fitting curves.
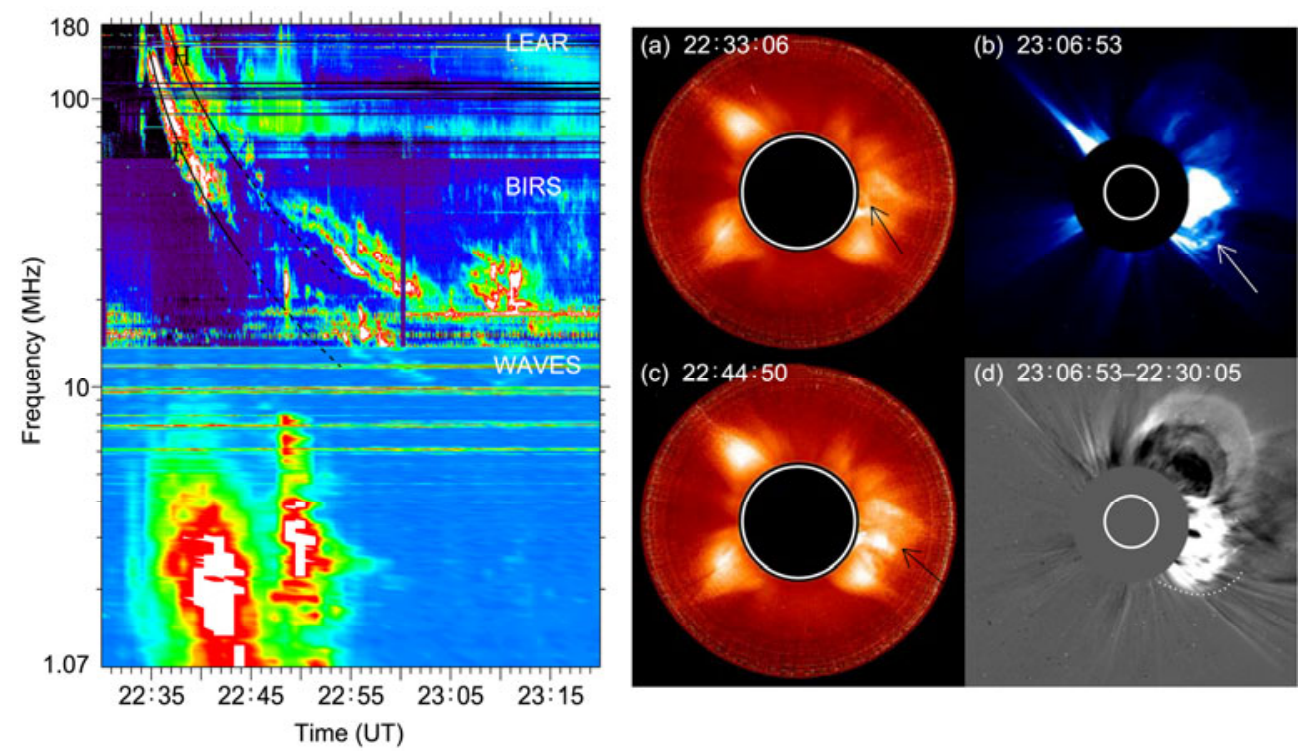

Figure 15 Left panel: Radio dynamic spectrum on 1 November 2003 from 22:30 UT to 23:20 UT, given by WAVES/WIND (1.07-13.8 MHz), BIRS $(13.8-62.5 \mathrm{MHz})$, and LEAR $(62.5-180 \mathrm{MHz})$. F and $\mathrm{H}$ denote the fundamental and harmonic branches. The two solid-dashed lines are spectral fittings before 22:44 UT using the one-fold Saito model and a shock speed of $900 \mathrm{~km} \mathrm{~s}^{-1}$. Right panel: (a)-(c) Coronal images observed by MK4 and C2. The arrows denote the CME fronts. (d) is the $\mathrm{C} 2$ difference image, in which the dotted curve plots the diffuse structure ahead of the bright ejecta. The $\mathrm{CME}$ erupts from a multi-streamer system with a bright arc front, pushing aside surrounding streamers during its lateral expansion (taken from [141]). 
A straightforward explanation of the spectral bump is that the radio-emitting shock was passing through a high density structure (presumably a streamer). Four images given by MK4 and LASCO C2 are shown in Figure 15. It can be seen that the CME erupts from a multi-streamer system with a bright arc front as denoted by the arrows. This front hits both streamer structures on its northern and southern flanks. From the $\mathrm{C} 2$ difference image shown in the figure, a diffuse structure is observed ahead of the bright ejecta. This feature is usally taken as the shock sheath structure [34]. To tell the interaction with which streamer accounting for the spectral bump, in Figure 16 ten basedifference images (MK4 images subtracted the pre-eruption image at 22:30 UT) for the period of 22:33-23:00 UT are shown.

To determine the physical cause of the spectral bump, we first examine carefully the CME interactions with both streamers during the type II burst. From Figure 16 and the corresponding movie, it can be seen that the expansion of the CME front is highly asymmetric. The shock wave encountered the northen streamer first at 22:39 UT 1.6 Rs, and the southern streamer gets deflected by the CME shock a few minutes later at 22:45 UT. The corresponding interaction regions are pointed out with arrows in Figure 16(c) and (e). Note that the spectral bump starts around 22:46 UT, temporally coincident with the start of deflection of the southeren streamer, yet several minutes later than the time when the northern streamer is first deflected.

In addition, Feng et al. [141] compared the shock heights measured along the two lines plotted in Figure 16 and those deduced from the type II emission using the Saito model. They found that the radio-fitting heights are more consistent with those along the dashed line, i.e. along the southern part of the CME front. To demonstrate that the Saito model is a reasonable assumption, Feng et al. [141] deduced the den- sity profiles with the pre-eruption $\mathrm{pB}$ data measured by MK4 along 3 PAs of $230^{\circ}, 240^{\circ}$, and $270^{\circ}$. All the obtained profiles are considerably close to the Saito model at distances below 1.6 Rs. Feng et al. also compared the transit time of the shock across the southern streamer and the bump duration. According to the dynamic spectrum, the bump lasts for $\sim 10 \mathrm{~min}$. Assuming the shock propagates with a speed of $900 \mathrm{~km} \mathrm{~s}^{-1}$ across a distance of $0.5-1$ Rs that is the approximate width of the southern streamer at $\sim 2.3 \mathrm{Rs}$, they found that it takes about $6-13$ min for the shock to cross the streamer. This agrees with the bump duration.

The above analyses indicate that the type II spectral bump is caused by the shock-radio source propagating across the southern streamer. It can be further deduced that the type II burst is emitted from the southern flank of the CME shock, corresponding to quasi-perpendicular shock geometry. A cartoon illustration of our understanding of the event is plotted in Figure 16. The timings and locations of the bump are given along with the estimated type II sources. Other radio signatures possibly associated with the CMEstreamer interaction are also shown [141].

(ii) A type II event with spectral break. CMEs frequently originate from inside of a streamer. The shock, if generated within the streamer, may propagate across the streamer boundary, at which the density drops sharply. This may affect the spectral shape of type II in a similar manner as that in the bump event. Kong et al. [142] reported one such event dated on 27 March, 2011.

The dynamic spectrum recorded by BIRS (10-62 MHz: Erickson [153]) and Learmonth (62-75 MHz) from 00:1000:50 UT is shown in Figure 17. The GOES X-ray flux of the acompanying C3.2 flare is also plotted, reaching its peak at $\sim 00: 14$ UT. Several type III bursts appear during $00: 13: 30-00: 16: 30$ UT. The type II burst starts at $\sim 00: 27$ UT, while the X-ray flux already relaxes to its background.
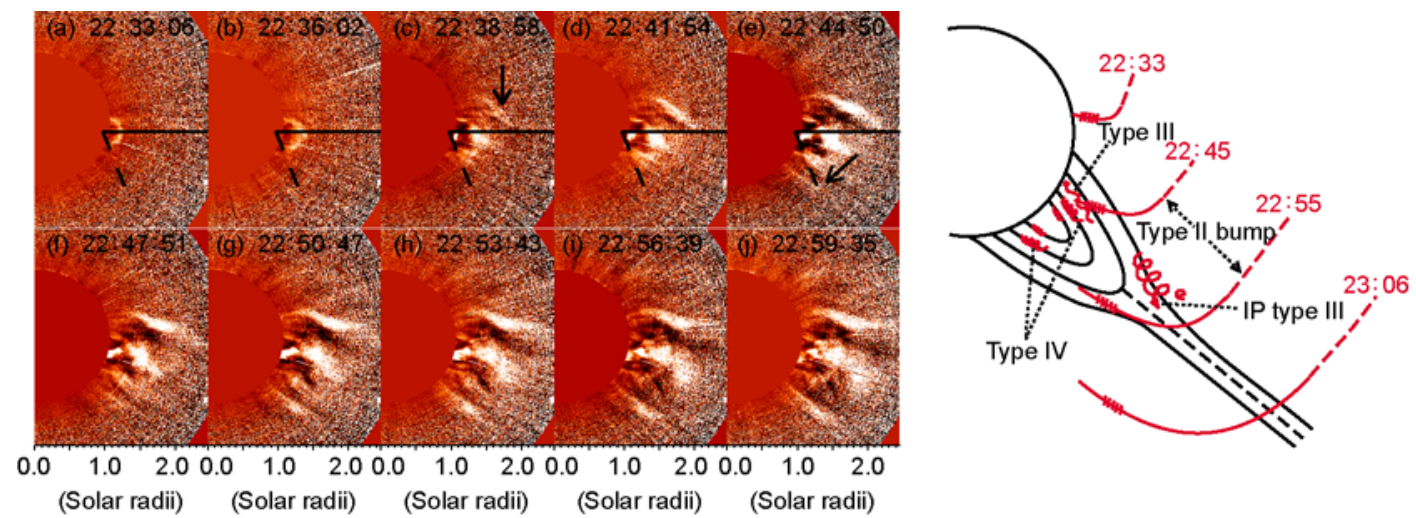

Figure 16 Left panels: Ten MK4 images for the 1 November 2003 event from 22:33:06 UT to 22:59:35 UT with three minutes apart. All images have subtracted the pre-eruption image at 22:30:06 UT. The solid and dashed lines in the upper panels are drawn to measure the heights of the CME fronts. Right panel: Schematic describing the streamer magnetic topology, the outward propagation of the shock, and the location of the type II spectral bump. The dashed line above the streamer cusp denotes the HCS. The estimated type II sources are shown as segments. The energetic electrons confined within streamers, and those flowing sunward and anti-sunward are indicated. These electrons are related to type IV, possible RS-III, and low-frequency type III bursts present in the dynamic spectrum (taken from [141]). 

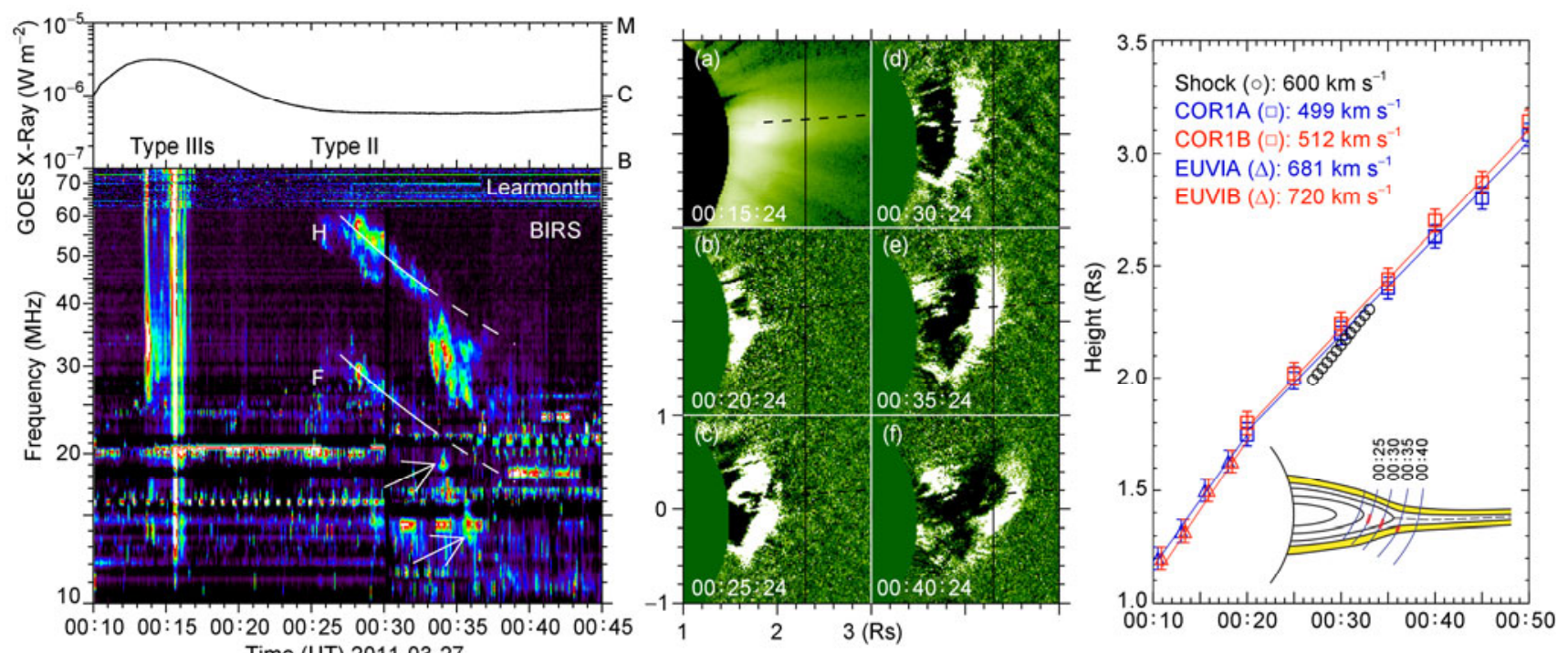

Figure 17 Left panel: The upper part plots the GOES soft X-ray flux, showing a C3.2 flare at the time of type IIIs onset. The lower part is the radio dynamic spectrum from BIRS (10-62 MHz) and Learmonth (62-75 MHz). "F" and " $\mathrm{H}$ " stand for the fundamental and harmonic branches. Solid lines are spectral fittings using the twofold Newkirk density model and a shock of $600 \mathrm{~km} \mathrm{~s}^{-1}$, the dashed line is the extension to the harmonic fitting. The two white arrows indicate the fundamental counterparts of the upper post-break emission. Middle and right panels: Left side are COR1B data between 00:15 and 00:40 UT, with FOVs of $2.0 \times 2.0$ Rs. (a) is the original image at 00:15 UT and the others are running difference images. The black dashed lines represent the center of the streamer, and the vertical black lines the estimated height of the steamer cusp. The right side plots the heights of the disturbance front measured from STEREO, with triangles for EUVI and squares for COR1 and in blue/red for STEREO A/B. The error bars indicate measurement uncertainties of the heights, estimated to be $\sim 0.05$ Rs. The lines are obtained by linearly fitting the EUVI and COR1 data points and the deduced propagation speeds are shown in the upper left corner. The fitted radio shock heights are also shown as open circles. The superposed caroon is the schematic diagram illustrating the physical origin of the type II burst, in which black and blue curves represent the streamer field lines and shock locations. The streamer boundary is shaded in yellow. The radio-emitting region is indicated by the red thick segment (taken from [142]).

This indicates that the type II burst is not caused by the flare-heating blast wave.

The type II starts from $\sim 60 \mathrm{MHz}$ with both $\mathrm{F}$ and $\mathrm{H}$ branches. The $\mathrm{H}$ branch is more pronounced with more recognizable details, taken as the focus of this study. The most prominent feature of this branch is the presence of a spectral break ( 00:33 UT, $\sim 43 \mathrm{MHz}$ ) which separates the whole spectrum into two parts. Before the break, the spectrum drifted gradually in 6 minutes from 60 to $43 \mathrm{MHz}$ with a mean drift rate of $\sim-0.05 \mathrm{MH} \mathrm{s}^{-1}$. This part of the spectrum can be well-fitted with a two-fold Newkirk density model [127]. The fitting gives a shock speed of $600 \mathrm{~km} \mathrm{~s}^{-1}$ and a shock height from 2.0-2.3 Rs. After the break, the spectrum continues with a much faster drift with frequencies declining from $\sim 43$ to $\sim 24 \mathrm{MHz}$ within 2-3 min. The average frequency drift after the break is $\sim-0.3 \mathrm{MHz} \mathrm{s}^{-1}$ about 6 times faster than its preceding counterpart. Therefore, it is not possible to fit the whole spectrum with a single density model and similar shock speed, neither is it possible to attribute the fast drift to the increase of the shock speed. The most viable explanation is that the fast drift is caused by a sudden density decrease at the radio source, as supported by the following analyses.

Kong et al. [142] examined the imaging data of the eruption obtained by COR1/COR2 and EUVI [154] onboard STEREO A and B and AIA [155] onboard SDO. The three spacecraft formed a "T" configuration with the event observed by SDO as a disk event and by STEREO as a limb event. This allows one to observe the details of the eruption source and measure the CME dynamics with minimum effect of projection, simultaneously. Figure 18(a)-(c) presents the coronal field configurations given by the PFSS model on the basis of HMI/SDO [156] measurements for CR2108. The field lines are adjusted to the viewing angles of the three spacecraft. The coronal images from COR1 and EUVI at about 00:10 UT are also shown in (a) and (c). The source of the event was NOAA AR11176, located at S16E04 (http://www.solarmonitor.org/) in the SDO view. The equatorial streamer corresponds to the large-scale closed field lines striding over ARs 11176 and 11177. Therefore, the eruption originates from one foot of the streamer. The vertical line in Figure 18 presents the estimated cusp location of the streamer at $\sim 2.3$ Rs.

In the middle panel of Figure 17 we present 6 running difference images of COR1B from 00:15-00:40 UT. The measured distances of the eruption front from the EUVI and COR1A/B observations are plotted together with the radioshock distances as given by fitting the slow-drift spectrum with the twofold Newkirk model. It can be seen that they are consistent with each other. As mentioned, the shock speed used in the radio fitting is $600 \mathrm{~km} \mathrm{~s}^{-1}$, also very close to the measured speeds of the disturbance front with EUVI 

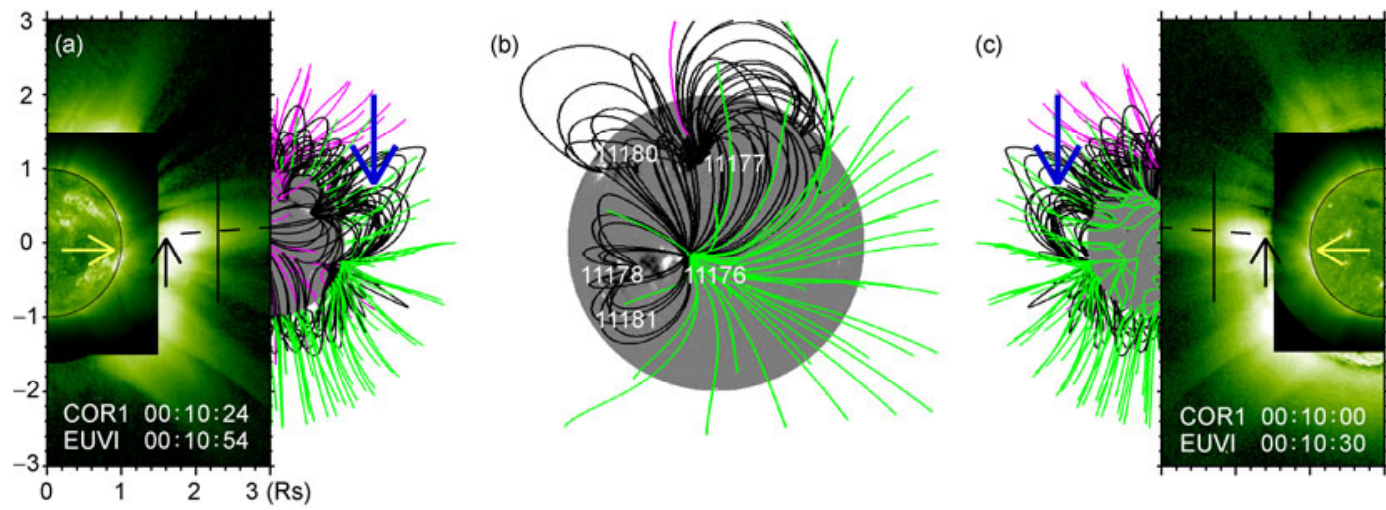

Figure 18 Magnetic field configurations given by the PFSS model based on the measurements with SDO/HMI for CR 2108 . The field lines have been adjusted to the individual views of the three spacecraft. The closed lines are colored in black, and the open inward (outward) field lines in purple (green). (a) and (c) also show coronal images from COR1 and EUVI $195 \AA$ at about 00: 10 UT with FOVs of 1.5×3.0 Rs and 3.0×6.0 Rs (taken from [142]).

and COR1 A (B), which are 681 and $499 \mathrm{~km} \mathrm{~s}^{-1}$ (720 and $512 \mathrm{~km} \mathrm{~s}^{-1}$ ), respectively. This means that the observed disturbance front represents the shock structure accouting for the type II radio burst.

Around the time of the spectral break ( 00:33 UT), the disturbance front is observed to propagate from $2.24 \mathrm{Rs}$ $(00: 30 \mathrm{UT})$ to 2.44 Rs $(00: 35 \mathrm{UT})$ passing by the vertical line, i.e. the estimated streamer cusp location. On the other hand, according to the spectral fitting, the spectral break $(\sim 43 \mathrm{MHz})$ corresponds to a shock at $\sim 2.3 \mathrm{Rs}$, also agreeing with the height of the streamer cusp.

With the above analyses, Kong et al. [142] concluded that the type II burst is generated as the CME shock propagating outward from inside of the streamer, the pre-break emission is excited within the streamer while the post-break spectrum is due to the shock crossing the streamer boundary. Therefore, the density gradient there can be estimated as follows. The H-branch frequency (density) decreases from $40 \mathrm{MHz}\left(5 \times 10^{6} \mathrm{~cm}^{-3}\right)$ to $25 \mathrm{MHz}\left(2 \times 10^{6} \mathrm{~cm}^{-3}\right)$ in $\sim 2$ minutes, within a short distance of 0.1 Rs (the shock speed is assumed to be $600 \mathrm{~km} \mathrm{~s}^{-1}$ ). This density gradient is for the streamer boundary at $\sim 2.3 \mathrm{Rs}$, which should be taken as a lower limit considering that the shock transit direction may not be perpendicular to the density gradient.

The process is further illustrated with the inset of Figure 17 , in which the streamer magnetic field lines and shock front locations at various times are drawn. The transition layer from the dense streamer to the surrounding dilute solar wind is colored in yellow. The type II radio source is indicated by the thick red segment. The shock front crossed the streamer cusp at a time between 00:30 UT and 00:35 UT. The spectral break was observed at 00:33 UT corresponding to the start of the transit of the radio source across the streamer boundary. The radiation ended soon after the radio sources moved out of the transition layer, perhaps because the shock weakened in the faster solar wind. Indeed, since the solar wind is less dense and the Alfvén speed is ex- pected to be faster, the shock wave inside the streamer may "unshock" in the solar wind and fail to accelerate enough electrons to excite type II bursts.

\section{Conclusions and discussion}

This paper presents a review on some recent research progresses on coronal dynamics, with most topics closely related to the studies carried out by the author and collaborators. It is therefore unavoidably biased by the author's personal point of view. In the following we first summarize the major scientific results according to their order of presence in the main text, then we show relevant unresolved issues and possible directions for future research.

In Section 1 we present recent numerical and observational studies on streamer morphologies, acceleration of streamerrelated slow solar wind, physical mechanism of persistent releases of streamer blobs, and the speed diagnostic results for the near-Sun slow wind.

White light (also the $\mathrm{pB}$ ) and UV data provide important clues to understanding the streamer morphologies. However, due to a lack of direct measurements of coronal magnetic field, it remains controversial regarding the magnetic topology underlying the streamer. The debates lie in whether the streamer helmet is fully-closed or partially-open, and whether it is bipolar or multi-polar magnetically. With a coronal and solar wind MHD model driven by the Alfvénic turbulence, it is shown that similar pB morphology is obtainable with either closed or fully-open magnetic configurations. Therefore, it is not straightforward to infer the magnetic topology of the streamer with the $\mathrm{pB}$ data. On the other hand of UV spectral studies, the deduced latitudinal distribution of OVI outflow speeds agrees with the scenario of the streamer helmet being a closed structure as a whole; In addition, with a multi-fluid MHD model considering one species of heavy ions (O ions or alpha particles) it was found that the surprising bifurcated fork-like structure 
observed in OVI lines can be understood from the distribution and motion of heavy ions during the evolution of a bipolar-closed streamer structure. The model predicted that the UV-fork structure appears several days after the streamer stabilization. Further studies are required to clarify both the magnetic topology and the nature of the streamer UV morphology $[15,45]$.

Although streamers are observed to be closely related to the origin and acceleration of the slow wind, it remains an open issue regarding how the streamer magnetic topology affects the slow wind dynamics. The flux tubes around streamers are featured by a special expanding process with an obvious curvature. Effects of the expansion have been extensively examined, yet few studies investigated the role of the tube curvature. Latest calculations reveal that the tube curvature has a significant influence on the solar wind acceleration with a larger curvature resulting in a slower solar wind. Follow-up studies with alpha particles also found that the proton-alpha particle differential speeds depend considerably on the curvature of the field. This study provides a natural physical explanation to the observed dependence of the solar wind speed on the angular distance from the tube root to nearby coronal hole $[57,59]$.

Streamer blobs are usually treated as velocity tracers of the slow wind along the plasma sheet. In this review persistent-release events of blobs were investigated. Measurements of subsequent blobs were used to infer the temporal variation of the slow wind speed at a fixed distance. It is found that the near-Sun slow wind speed is featured by a large variability implicating that the similar characteristic discovered in the slow wind in-situ may have its origin near the Sun. This is the first time that such velocity variation of the near-Sun slow wind is deduced observationally. With a MHD model of the corona and solar wind it is found that the streamer can develop an intrinsic MHD instability process due to the failure of plasma confinement at the streamer cusp. The repeated occurrence of the instability and associated reconnections account for the persistent releases of streamer blobs. This mechanism of blob generation explains well the quasi-periodic release of streamer blobs [43,62].

In Section 2, we present recent studies on energy release mechanisms of CMEs with a flux-rope MHD model, studies on magnetic islands along the CME current sheet, and the first-time observation of magnetic island coalescence and associated electron accelerations in space.

The energy released during a CME is mainly from the coronal magnetic field, yet it remains elusive regarding how the energy is released and converted into the CME kinetic energy. With a novel numerical flux rope model in the presence of the solar wind it is investigated of the two means of magnetic energy releases, the large-scale ideal MHD flux rope instability and the magnetic reconnection process across local current sheet structure. Force balance analyses indicate that the most important driving force of the eruption stems from the flux rope self force. This is the major force component through which the flux rope instability converts the magnetic energy into the CME kinetic energy. During the eruption, an important sunward pulling force on the rope is exerted by the current sheet that is formed during the eruption, reconnections across which greatly weaken the pulling force. This results in a further release of magnetic energy into kinetic energy through the apparently-enhanced self force. This is suggested to be the major mechism of how reconnections induce CME accelerations. Modeling results indicate that fast CMEs can be produced even in the absence of magnetic reconnection, and a smooth transition from fast to slow CMEs can be observed when varying the background field strength. This means that the two types of CMEs may be physically identical. The observed three-phase acceleration of CMEs is also reproduced [29,92,93].

Current sheet structures are present during a CME and atop a helmet streamer, reconnections across which may produce magnetic islands or plasma blobs, which are observable with coronagraphs as bright structures. Therefore, properties of magnetic reconnections can be inferred through measurements of the blob dynamics. Comparing the velocity distributions of CME- and streamer-blobs, it is found that two physically-different reconnection processes are present in the outer corona. The differences lie in the dynamics of the reconnection products (i.e. blobs), magnetic field structures and strengths at the reconnection region, as well as the reconnection-triggering processes [70].

It is important to understand how electrons are energized during reconnections of a solar eruption. Previous theoretical studies indicate that coalescence of magnetic islands during reconnections can accelerate electrons efficiently. However, the theory received no observational support. In this study making use of coronagraph data on board the twin STEREO spacecraft, we report observations of macroscopic magnetic island coalescence along a CME-trailing current sheet. Type-III like radio burst associated with the coalescing process is found, indicating efficient electron acceleration during the process. This is the first-time report of magnetic island coalescing process observed in space. This study provides support to the theory of electron acceleration by magnetic island coalescence during reconnection [37].

In Section 3 we focus on white-light and radio manifestations of CME-streamer interactions, including the streamer wave phenomenon in the aftermath of CME-caused streamer deflections, the associated coronal seismological studies, the spectral bump and break features of type II radio bursts as a result of shocks crossing dense streamers, and the diagnostic results of type II source properties.

Waves represent the fundamental responses of a plasma system to disturbances. It is natural to ask whether the longprotruding streamer structure can exhibit wave-like oscillation upon disturbances of nearby eruptions. This work provides a positive answer to this question by discovering the streamer wave phenomenon propagating along the plasma 
sheet structure in the aftermath of CME-streamer interaction. It is one of the largest wave phenomena ever found in the corona. A novel coronal seismological method has been developed with the measurements of the wave dynamics and available observational constraints on the solar wind outflow speed and electron densities. The mothod can be used to dianose the variations of the Alfvén speed and magnetic field strength from 3-10 Rs along the streamer plasma sheet. This study inspires further theoretical investigations on MHD wave modes supported by magnetized shear flows [138-140,157].

It remains controversial regarding the source properties of solar type II radio bursts. In this series of studies, we propose to diagnose the source location and relevant shock geometry by relating the specific features of type II spectral shape with eruptive processes recorded by solar imaging instruments. Two spectral features are defined as the sepctral bump and spectral break that are interpreted to be caused by the radio source (presumably a part of the CME shock) propagating across a dense streamer structure from either outside or inside of the streamer. The corresponding type II source locations and the shock geometry are then inferred from the observed CME-streamer interaction process. This study provides a novel approach of diagnosing the type II source properties [141,142].

The scientific results presented in this paper have been summarized above, in the following we show relevant unresolved issues and possible directions for future research.

Topology and dynamics of coronal streamers, origin and acceleration of the slow solar wind, as well as their physical connections are long-standing unresolved issues in solar and space physics. It is generally believed that the streamer region represents one important source of the slow wind. Other possible sources of the slow wind have been reported to be the border of polar coronal holes, inside of small coronal holes, and active regions. It remains as an open issue regarding how to observationally discern and differentiate the solar wind plasmas from various sources, and how to set up the physical connection between the in-situ measured plasma parameters and the remotely-sensed plasma and magnetic structures on the solar surface and in the corona.

Recent coronagraph observations reveal a new type of streamer structure, the pseudo-streamer (PS) which consists of a pair of loop arcades separating coronal holes of the same polarity. The plasma sheet is unipolar and not associated with a current sheet [158]. This structure is also considered to be an important source of the solar wind. It remains un-answered regarding its MHD equilibrium and instabilities, the formation mechanism of its plasma sheet, the origin and acceleration of relevant solar wind, and its association with CME origins.

Besides remote-sensing observations of the corona, another important approach to studying coronal dynamics is to search through in-situ data for coronal imprints on the solar wind. By analyzing the elemental composition, charge state distribution, and structure details in the solar wind, one can infer the counterpart structure properties and physical processes in the corona. For example, the in-situ detection of streamer blobs can be used to diagnose properties of their sources, i.e. the upper closed region of the streamer $[10,43,159]$. This is also important to our understanding of the streamer instability and the slow wind origin. A study on in-situ blobs has been presented by Rouillard et al. [160], however, no elemental and charge state analyses were conducted. In addition, by finding in-situ counterpart of coronal structures, it is possible to settle the debate on the physical nature of the bright ray behind a CME.

A significant part of the studies reviewed here can be connected with magnetic reconnections. Reconnections play a crucial role during solar eruptions, which are able to rapidly convert magnetic energy into kinetic and thermal energies of plasmas (and energetic particles). Although coronal magnetic fields are not directly measurable, they can be inferred indirectly through spectral and imaging data. Examples presented here are studies on streamer and CME blobs, whose dynamics are measured and compared to infer physical properties of accounting magnetic reconnections.

One relevant open question is how electrons are accelerated during reconnections. A first-time observation of electron accelerations associated with coalescence of magnetic islands was reviewed. However, the process took place in the outer corona far away from the flare reconnection region. As indicated by observations, about $10 \%-50 \%$ of the energy released during a large flare is carried by accelerated electrons $>20 \mathrm{keV}[161,162]$. Therefore, it is rather important to answer how electrons are accelerated, and in a more specific manner, what roles played by island coalesence or contraction in electron acceleration during a flare reconnection. Further studies are required to resolve this issue.

Reconnections play an important role in the rapid release of magnetic energy into CME kinetic energy. However, it is not the only important process that contributes to CME accelerations. And there is no concensus regarding how CMEs are accelerated via reconnection. Some authors suggest that the CME ejecta (presumably a flux rope system) is accelerated mainly through its dynamical coupling with the highspeed reconnection exhausts [28]. It is known that the exhausts move at a speed lower than the local Alfvén speed. Therefore, with the above scenario it is not easy to understand the acceleration of super-Alfvénic fast CMEs and also that of CMEs with significant acceleration after the major flaring phase. In addition, it is also questionable regarding whether the kinetic energies carried by reconnection exhausts are enough to provide significant accelerations of the whole ejecta.

With the numerical flux rope model for CMEs, it is shown that the eruption is energized by the free energy released during the development of the flux rope instability, and the magnetic to kinetic energy conversion is mainly through the work done by the flux rope self force. Part of 
the free energy, not releasable via the ideal-MHD flux rope instability, is contained in the current sheet fromed during the eruption. Once the resistive reconnection sets in, this part of free energy is further released. This greatly weakens the corresponding retarding force acting on the flux rope and consequently the rope self force is able to convert more magnetic energy into kinetic energy. This has been suggested to be the major mechanism of reconnection-induced CME acceleration in a flux rope system [29,93]. Observationally, it is still very difficult to tell the relative contributions of different energy release processes with available datasets.

This paper also reviews the latest progresses on CMEstreamer interactions as observed in the white light and radio data, including studies on streamer waves, type-II spectral bump, and type-II spectral break. To reveal excitation conditions of streamer waves, Feng et al. [140] found 8 candidate events throughout SC 23, and defined their common observational features. This provides important clues to understanding the wave excitation conditions. However, more events should be analyzed and MHD modelings of CMEstreamer interactions should be conducted for a deeper understanding of this phenomena.

For the type II events reviewed here, the radio-emitting source properties and corresponding shock geometry can be inferred using the mentioned spectral features and their established physical connections with CME-streamer interactions. With the spectral-bump feature, the type II source is found to be along the flank of the CME shock with a quasiperpendicular geometry. Because of the spectral elevation, which corresponds to the entering process of the radio source into a denser structure, is completed within a relatively short time, the size of the radio source is estimated to be less than $\sim 0.05 \mathrm{Rs}$ and much smaller than the width of the streamer structure [163]. This reveals a point-like source of type-II burst. With the spectral-break feature, it is concluded that the pre-break slow-drift emission is generated within the streamer and the post-break fast-drift emission corresponds to the shock propagating across the streamer boundary. Of course, these deductions of the type II sources require further verification using with future high-resolution radio imaging data at relevant frequencies.

Besides the above phenomena, CME-streamer interactions may have other manifestations observable with present instruments. For example, reconnections may occur at the CME-streamer interaction region as long as the magnetic fields of CMEs and streamers are appropriately aligned. CMEs can also trigger and drive reconnections along the streamer current sheet which may generate blob structures observable with coronagraphs. Investigations into these structures are helpful to understanding the CME-streamer reconnections and associated physical consequences.

In addition, it should be noted that the streamer is composed of high-density plasmas with negligible outflow velocity and relatively low Alfvén speed. These streamer properties, especially the low characteristic speed, favor the formation and enhancement of coronal shocks. At the same time, the closed magnetic topology, the cusp and the current-sheet structures of the streamer may play a role in the shock-related physics as will be further discussed in the following paragraphs. The major observational manifestion of coronal shocks is type II radio burst. Therefore, future studies should continue to combine spectral and imaging observations to further understand the physics of coronal shocks and their consequences.

For those CMEs originating from the streamer, the CMEdriven shock may be generated within the streamer. The propagation of the shock through the closed streamer field lines gives rise to a magnetic collapsing geometry being an efficient electron accelerator [164-166]. This geometry, representative of a shock-enhanced magnetic mirror system, can effectively confine electrons, and return them to the shock for repeated acceleration. This may have important effects on coronal electron accelerations and corresponding radio bursts in the metric wavelength, and should be clarified in future observational studies. Theoretical studies on the role of streamers in shock-induced electron acceleration and type II radio bursts should also be pursued.

Due to the special plasma-magnetic conditions in the streamer region, double shocks that are separated from each other may be driven by a single eruption at places inside and outside the streamer. In addition, during an eruption multi-reconnections may take place along different current sheets that are possibly located behind the CME, along the CME-streamer interaction region, and atop the streamer. It remains unknown whether the double-shocks and multi-reconnections can be observed, and how they may contribute to particle acceleration and plasma turbulence in the corona. During the forthcoming maximum and declining phases of solar activities, more events of CME-streamer interactions will occur which should be further examined with present and future multi-wavelength data from multi vantage points for a better understanding of the physics involved.

I thank the editor for the invitation to prepare this review. This work would be impossible without the intimate collaborations and helpful discussions with my colleagues and graduate students. Special thanks to Wang Bin for his help in preparing the manuscript, and Dr. Li Bo and Song HongQiang for useful discussions. I am also grateful to the SOHO/STEREO/SDOI WIND, and BIRS/Learmonth teams for making their high-quality scientific data available for us. This work was supported by the National Natural Science Foundation of China (40825014, 40890162, 41028004 and 41274175), and the National Basic Research Program of China (2012CB825601).

1 Brueckner G E, Howard R A, Koomen, et al. The large angle spectroscopic coronagraph (LASCO). Sol Phys, 1995, 162: 357-402

2 Schatten K H, Wilcox J M, Ness N F. A model of interplanetary and coronal magnetic fields. Sol Phys, 1969, 6: 442-455

3 Schrijver C J, De Rosa M L. Photospheric and heliospheric magnetic fields. Sol Phys, 2003, 212: 165-200

4 Pneuman G W, Kopp R A. Gas-magnetic field interactions in the so- 
lar corona. Sol Phys, 1971, 18: 258-270

5 Koutchmy S, Livshits M. Coronal streamers. Space Sci Res, 1992, 61: 393-417

6 Feldman W C, Asbridge J, Bame S, et al. The solar origins of solar wind interstream flows: Near-equatorial coronal streamers. J Geophys Res, 1981, 86: 5408-5416

7 Gosling J T, Borrini G, Asbridge J R, et al. Coronal streamers in the solar wind at 1 AU. J Geophys Res, 1981, 86: 5438-5448

8 Woo R, Martin J M. Source regions of the slow solar wind. Geophys Res Lett, 1997, 24: 2535-2538

9 Habbal S R, Woo R, Fineschi S, et al. Origins of the slow and the ubiquitous fast solar wind. Astrophys J, 1997, 489: L103-L106

10 Wang Y M, Sheeley N R, Socker D J, et al. The dynamical nature of coronal streamers. J Geophys Res, 2000, 105: 25133-25142

11 Cuperman S, Ofman L, Dreyer M. Thermally conductive MHD flows in helmet-streamer coronal structures. Astrophys J, 1990, 350: 846-855

12 Wang Y M. Two types of slow solar wind. Astrophys J, 1994, 437: L67-L70

13 Chen Y, Hu Y Q. A two-dimensional Alfvén-wave-driven solar wind model. Sol Phys, 2001, 199: 371-384

14 Chen Y, Esser R, Strachan L, et al. Stagnated outflow of $\mathrm{O}^{+5}$ ions in the source region of the slow solar wind at solar minimum. Astrophys J, 2004, 602: 415-421

15 Li B, Li X, Labrosse N. A global 2.5-dimensional three fluid solar wind model with alpha particles. J Geophys Res, 2006, 111: A08106

16 Sheeley Jr N R, Wang Y M, Hawley S H, et al. Measurements of flow speeds in the corona between 2 and 30 Rs. Astrophys J, 1997, 484: 472-478

17 Sheeley Jr N R, Wang Y M. In/Out Pairs and the detachment of coronal Streamers. Astrophys J, 2007, 655: 1142-1156

18 McAllister A H, Hundhausen A J. Solar Driver of Interplanetary Disturbances. San Francisco, Calif: The Astronomical Society of The Pacific, 1996. 171

19 Subramanian P, Rich N B, Howard R A. The relationship of coronal mass ejections to streamers. J Geophys Res, 1999, 104: 22321-22330

20 Howard R A, Sheeley N R Jr, Michels D J, et al. Coronal mass ejections-1979-1981. J Geophys Res, 1985, 90: 8173-8191

21 Hundhausen A J. Sizes and locations of coronal mass ejections: SMM Observations from 1980 and 1984-1989. J Geophys Res, 1993, 98: $13177-13200$

22 Bemporad A, Sterling A C, Moore R L, et al. A new variety of coronal mass ejection: Streamer puffs from compact ejective flares. Astrophys J, 2005, 635: 189-192

23 Crooker N, Joselyn J A, Feynman J. Coronal Mass Ejections. Washington: Geophysics Monography Series, 1997. 99

24 Forbes T G, Lin J. What can we learn about reconnection from coronal mass ejections? J Atmosph Solar-Terrest Phys, 2000, 62: 14991507

25 Low B C. Coronal mass ejections, magnetic flux ropes, and solar magnetism. J Geophys Res, 2001, 106: 25141-25163

26 Benz A O. Flare observations. Living Rev Sol Phys, 2008, 5: 1

27 Webb D F, Howard T A. Coronal mass ejections: Observations. Living Rev Sol Phys, 2012, 9: 3

28 Chen P F. Coronal mass ejections: Models and their observational basis. Living Rev Sol Phys, 2011, 8: 1

29 Chen Y, Hu Y Q, Xia L D. Two energy release processes for CMEs: MHD catastrophe and magnetic reconnection, Adv Space Res, 2007, 40: $1780-1786$

30 Thompson B J, Plunkett S P, Gurman J B, et al. SOHO/EIT observations of an Earth-directed coronal mass ejection on May 12, 1997. Geophys Res Lett, 1998, 25: 1999-2002

31 Hudson H S, Webb D F. Soft X-ray signatures of coronal ejections. In: Crooker N, Joselyn J A, Feynman J, et al., eds. Collection in Coronal Mass Ejections. Washington: Geophysics Monography Series, 1997. 99

32 Nelson G J, Melrose D B. Type II bursts. In: McLean D J, Labrum N R, eds. Solar Radiophysics. Cambridge: Cambridge University Press, 1985. 333

33 Sime D, Hundhausen A. The coronal mass ejection of July 6, 1980: A candidate for interpretation as coronal shock wave. J Geophys Res, 1987, 92: 1049-1055

34 Vourlidas A, Wu S T, Wang A H, et al. Direct detection of a coronal mass ejection-associated shock in large angle and spectrometric coronagraph experiment white-light images. Astrophys J, 2003, 598: 1392-1402

35 Reames D V. Particle acceleration at the sun and in the heliosphere. Space Sci Rev, 1999, 90: 413-491

36 Sheeley N R, Hakala W N, Wang Y M. Detection of coronal mass ejection associated shock waves in the outer coronal. J Geophys Res, 2000, 105: 5081-5092

37 Song H Q, Chen Y, Li G, et al. Coalescence of macroscopic magnetic islands and electron acceleration from STEREO observation. Phys Rev X, 2012, 2: 021015

38 Zurbuchen T H, Steiger R V, Gruesbeck J, et al. Sources of solar wind at solar minimum: Constraints from composition data. Space Sci Rev, 2012, 172: 41-55

39 Schwenn R, Inhester B, Plunkett S P. et al. First view of the extended green-line emission corona at solar activity minimum using the LascoC1 coronagraph on SOHO. Sol Phys, 1997, 175: 667-684

40 Banaszkiewicz M, Axford W I, McKenzie J F. An analytic solar magnetic field model. Astron Astrophys, 1998, 337: 940-944

41 Guhathakurta M, Sittler E C, Ofman L. Semiempirically derived heating function of the corona heliosphere during the Whole Sun Month. J Geophys Res, 2006, 111: A11215

42 Wang S, Liu Y F, Zheng H N. Magnetic reconnection in multiple heliospheric current sheets. Sol Phys, 1997, 173: 409-426

43 Chen Y, Li X, Song H Q, et al. Intrinsic instability of coronal streamers. Astrophys J, 2009, 691: 1936-1942

44 Noci G. The quiescent corona and slow solar wind. Fifth SOHO Workshop: The corona and solar wind near minimum activity. In: Wilson A, ed. European Space Agency, 1997. 75-84

$45 \mathrm{Li} \mathrm{B}, \mathrm{Li} \mathrm{X}, \mathrm{Yu} \mathrm{H}$. Interpretation of the coronal magnetic field configuration of the Sun. Res Astron Astrophys, 2012, 12: 1693-1700

46 Raymond J C, Kohl J L, Noci G, et al. Composition of coronal streamers from the $\mathrm{SOHO}$ ultraviolet coronagraph spectrometer. Sol Phys, 1997, 175: 645-665

47 Akinari N. Morphological study of quiescent streamers during solar minimum by ultraviolet emission lines. Astrophys J, 2007, 668: 1196-1209

48 Strachan L, Suleiman R, Panasyuk A V, et al. Empirical densities, kinetic temperatures, and outflow velocities in the equatorial streamer belt at solar minimum. Astrophys J, 2002, 571: 1008-1014

49 Ofman L, Abbo L, Giordano S. Multi-fluid model of a streamer at solar minimum and comparison with observations. Astrophys J, 2011, 734: 30

50 McComas D J, Barraclough B L, Funsten H O, et al. Solar wind observations over Ulysses' first full polar orbit. J Geophys Res, 2000, 105: 10419-10434

51 McComas D J. Weaker solar wind from the polar coronal holes and the whole Sun. Geophys Res Lett, 2008, 35: L18103

52 Cranmer S R. Coronal holes. Living Rev Sol Phys, 2009, 6: 3

53 Chen Y, Hu Y Q. Effect of flow tube geometry on solar wind properties. Astrophys Space Sci, 2002, 282: 447-460

54 Wang Y M, Sheeley N R. Solar wind speed and coronal flux-tube expansion. Astrophys J, 1990, 355: 726-732

55 Arge C N, Luhmann J G, Odstrcil D, et al. Stream structure and coronal sources of the solar wind during the May 12th, 1997 CME. J Atmosph Solar-Terrestrial Phys, 2004, 66: 1295-1309

56 Hu Y Q, Habbal S R, Chen Y, et al. Are coronal holes the only source of fast solar wind at solar minimum? J Geophys Res, 2003, 108: 1377

57 Li B, Xia L D, Chen Y. Solar winds along curved magnetic field lines. Astron Astrophys, 2011, 529: A148

58 Leer E, Holzer T E. Energy addition in the solar wind. J Geophys Res, 1980, 85: 4681-4688

$59 \mathrm{Li} \mathrm{B}$, Chen Y, Xia L D. What geometrical factors determine the in situ solar wind speed? Chin Sci Bull, 2012, 57: 1409-1414

60 Forsyth R J, Marsch E. Solar origin and interplanetary evolution of 
stream interfaces. Space Sci Rev, 1999, 89: 7-20

61 Wang Y M, Sheeley N R, Walters J H, et al. Origin of streamer material in the outer corona. Astrophys J, 1998, 498: L165-L168

62 Song H Q, Chen Y, Liu K, et al. Quasi-periodic releases of streamer blobs and velocity variability of the slow solar wind near the sun. Sol Phys, 2009, 258: 129-140

63 Li X, Habbal S R, Kohl J L, et al. The effect of temperature anisotropy on observations of Doppler dimming and pumping in the inner corona. Astrophys J, 1998, 501: L133-L137

64 Cranmer S R, Kohl J L, Noci G, et al. An empirical model of a polar coronal hole at solar minimum. Astrophys J, 1999, 511: 481-501

65 Grail R R, Coles W A, Klinglesmith M T, et al. Rapid acceleration of the polar solar wind. Nature, 1996, 379: 429-432

66 Breen A R, Mikic Z, Linker J A, et al. Interplanetary scintillation measurements of the solar wind during whole sun month: Comparisons with coronal and in situ observations. J Geophys Res, 1999, 104: 9847-9870

67 Lee L C, Wang S, Wei C Q, et al. Streaming sausage, kink and tearing instabilities in a current sheet with applications to the Earth's magnetotail. J Geophys Res, 1988, 93: 7354-7365

68 Wang S, Lee L C, Wei C Q. Streaming tearing instability in the current sheet with a super-Alfvénic flow. Phys Fluids, 1988, 31: 1544-1548

69 Lapenta G, Knoll D A. Effect of a converging flow at the streamer cusp on the genesis of the slow solar wind. Astrophys J, 2005, 624: 1049-1056

70 Song H Q, Kong X L, Chen Y, et al. A statistical study on the morphology of rays and dynamics of blobs in the wake of coronal mass ejections. Sol Phys, 2012, 276: 261-276

71 Burlaga L F, Sittler E, Mariani F, et al. Magnetic loop behind an interplanetary shock: Voyager, Helios, IMP 8 observations. J Geophys Res, 1981, 86: 6673-6684

72 Lepping R P, Jones J A, Burlaga L F. Magnetic field structure of interplanetary magnetic clouds at 1 AU. J Geophys Res, 1990, 95: 11957-11965

73 Hu Q, Sonnerup B U O. Reconstruction of magnetic clouds in the solar wind: Orientations and configurations. J Geophys Res, 2002, 107: 1142

74 Lynch B J, Gruesbeck J R, Zurbuchen T H, et al. Solar cycle-dependent helicity transport by magnetic clouds. J Geophys Res, 2005, 110: A08107

75 Hundhausen A J. The many faces of the Sun: A summary of the results from NASA's solar maximum mission. In: Strong K T, Saba J L R, Haisch B M, et al., eds. Coronal Mass Ejections. New York: Springer, 1999. 143

76 Low B C, Hundhausen J R. Magnetostatic structures of the solar corona. II. The magnetic topology of quiescent prominences. Astrophys J, 1995, 443: 818-836

77 Forsyth R J, Bothmer V, Cid C. ICMEs in the inner heliosphere: Origin, evolution and propagation effects. Space Sci Rev, 2006, 123: $383-416$

78 Canfield R C, Hudson H S, McKenzie D E. Sigmoidal morphology and eruptive solar activity. Geophys Res Lett, 1999, 26: 627-630

79 Yan Y H, Deng Y Y, Karlicky M, et al. The Magnetic rope structure and associated energetic processes in the 2000 July 14 solar flare. Astrophys J, 2001, 551: L115-L119

80 Cheng X, Zhang J, Liu Y, et al. Observing flux rope formation during the impulsive phase of a solar eruption. Astrophys J, 2011, 732: L25

81 Zhang J, Cheng X, Ding M D. Observation of an evolving magnetic flux rope before and during a solar eruption. Nat Commun, 2012, 3: 747

82 Zhang J, Liu Y. Ubiquitous rotating network magnetic fields and extreme-ultraviolet cyclones in the quiet sun. Astrophys J, 2011, 741: L7

83 Li X, Morgan H, Leonard D, et al. A solar tornado observed by AIA/SDO: rotational fllow and evolution of magnetic helicity in a prominence and cavity. Astrophys J, 2012, 752: L22

84 Böhm S W, Scullion E, Steiner O, et al. Magnetic tornadoes as energy channels into the solar corona. Nature, 2012, 486: 505-508

85 Forbes T G, Isenberg P A. A catastrophe mechanism for coronal mass ejections. Astrophys J, 1991, 373: 294-307

86 Hu Y Q, Li G Q, Xing X Y. Equilibrium and catastrophe of coronal flux ropes in axisymmetrical magnetic field. J Geophys Res, 2003, 108: 1072

87 Fan Y, Gibson S E. Onset of coronal mass ejections due to loss of confinement of coronal flux rope. Astrophys J, 2007, 668: 1232-1245

88 Török T, Kliem B. Confined and ejective eruptions of kink-unstable flux ropes. Astrophys J, 2005, 630: L97-L100

89 Kliem B, Török T. Torus instability. Phys Rev Lett, 2006, 96: 255002

90 Mikic Z, Linker J A. Disruption of coronal magnetic field arcades. Astrophys J, 1994, 430: 898-912

91 Antiochos S K, DeVore C R, Klimchuk J A. A model for solar coronal mass ejections. Astrophys J, 1999, 510: 485-493

92 Chen Y, Li G Q, Hu Y Q. Force balance analysis of a coronal magnetic flux rope in equilibrium or eruption. Astrophys J, 2006, 649: 1093-1099

93 Chen Y, Hu Y Q, Sun S J. Catastrophic eruption of magnetic flux rope in the corona and solar wind with and without magnetic reconnection. Astrophys J, 2007, 665: 1421-1427

94 Chen J. Effects of toroidal forces in current loops embedded in a background plasma. Astrophys J, 1989, 338: 453-470

95 Krall J, Chen J, Santoro R. Drive mechanisms of erupting solar magnetic flux ropes. Astrophys J, 2000, 539: 964-982

96 Sheeley N R, Walters J, Wang Y M, et al. Continuous tracking of coronal outflows: Two kinds of coronal mass ejections. J Geophys Res, 1999, 104: 24739-24767

97 Andrews M D, Howard R A. A two-type classification of LASCO coronal mass ejection. Spsce Sci Rev, 2001, 95: 147-163

98 Yurchyshyn V, Yashiro S, Abramenko V, et al. Statistical distributions of speed of coronal mass ejections. Astrophys J, 2005, 619: 599-603

99 Vršnak B, Sudar D, Rudjak D. The CME-flare relationship: Are there really two types of CMEs? Astron Astrophys J, 2005, 435: 11491157

100 Zhang J, Dere K P. A statistical study of main and residual accelerations of coronal mass ejections. Astrophys J, 2006, 649: 1100-1123

101 Carmichael H. AAS-NASA Symposium on solar flares. Hess W N, ed. (NASA Sp-50), 1964. 451

102 Sturrock P A. Model of the high-energy phase of solar flares. Nature, 1966, 211: 695-697

103 Hirayanma T. Theoretical model of flares and prominences. I: Evaporating flare model. Sol Phys, 1974, 34: 323-338

104 Kopp R A, Pneuman G W. Magnetic reconnection in the corona and the loop prominence phenomenon. Sol Phys, 1976, 50: 85-98

105 Lin J, Forbes T G. Effects of reconnection on the coronal mass ejection process. J Geophys Res, 2000, 105: 2375-2392

106 Ciaravella A, Raymond J C, Li J, et al. Elemental abundances and post-coronal mass ejection current sheet in a very hot active region. Astrophys J, 2002, 575: 1116-1130

107 Webb D F, Burkepile J, Forbes T G, et al. Observational evidence of new current sheets trailing coronal mass ejections. J Geophys Res, 2003, 108: 1440

108 Ko Y K, Raymond J C, Lin J, et al. Dynamical and physical properties of a post-coronal mass ejection current sheet. Astrophys J, 2003, 594: 1068-1084

109 Lin J, Ko Y K, Sui L, et al. Direct observations of the magnetic reconnection site of an eruption on 2003 November 18. Astrophys J, 2005, 622: 1251-1264

110 Bemporad A, Poletto G, Suess S T, et al. Current sheet evolution in the aftermath of a CME event. Astrophys J, 2006, 638: 1110-1128

111 Vršnak B, Poletto G, Vujić E, et al. Morphology and density structure of post-CME current sheets. Astron Astrophys, 2009, 499: 905-915

112 Kohl J L, Esser R, Gardner L D, et al. The ultraviolet coronagraph spectrometer for the solar and heliospheric observatory. Sol Phys, 1995, 162: 313-356

113 Litvinenko Y E. Particle acceleration in reconnecting current sheets with a nonzero magnetic field. Astrophys J, 1996, 462: 997-1004

114 Wood P, Neukirch T. Electron acceleration in reconnecting current sheets. Sol Phys, 2005, 226: 73-95

115 Lin J, Ko Y K, Raymond J C. Investigation of thickness and electrical resistivity of the current sheets in solar eruptions. Astrophys J, 
2009, 693: 1666-1677

116 Liu Y, Luhmann J G, Lin R P, et al. Coronal mass ejections and global coronal magnetic field reconfiguration. Astrophys J, 2009, 698: L51-L55

117 Winterhalter D, Smith E, Burton M, et al. The heliospheric plasma sheet. J Geophys Res, 1994, 99: 6667-6680

118 Ko Y K. Modeling UV and X-ray emission in a post-coronal mass ejection current sheet. Astrophys J, 2010, 722: 625-641

119 Oka M, Phan T D, Krucker S, et al. Electron acceletration by multiisland coalescence. Astrophys J, 2010, 714: 915-926

120 Drake J F, Swisdak M, Che H, et al. Electron acceleration from contracting magnetic islands during reconnection. Nature, 2006, 443: 553-556

121 Fu X R, Lu Q M, Wang S. The process of electron acceleration during collisionless magnetic reconnection. Phys Plasmas, 2006, 13, 012309

122 Chen L J, Bhattacharjee A, Puhl-Quinn P A, et al. Observation of Energetic Electrons within Magnetic Islands. Nat Phys, 2007, 4: 19-23

123 Wang R S, Lu Q M, Du A M, et al. In situ observations of a secondary magnetic island in an ion diffusion region and associated energetic electrons. Phys Rev Lett, 2010, 104175003

124 Lu Q M, Wang R S, Xie J L, et al. Electron dynamics in collisinless magnetic reconnection, Chin Sci Bull, 2011, 56: 1174-1181

125 Kaiser M L, Kucera T A, Davila J M, et al. The STEREO mission: An introduction. Space Sci Rev, 2007, 136: 5-16

126 Bougeret J L, oetz K, Kaiser M L, et al. S/WAVES: The radio and plasma wave investigation on the STEREO Mission. Space Sci Rev, 2008, 136: 487-528

127 Newkirk G Jr. The solar corona in active regions and the thermal origin of the slowly varying component of solar radio radiation. Astrophys J, 1961, 133: 983-1013

128 Bemporad A, Poletto G, Landini F, et al. Magnetic reconnection processes induced by a CME expansion. Ann Geophys, 2008, 26: 3017-3024

129 Bemporad A, Soenen A, Jacobs C, et al. Side magnetic reconnections induced by coronal mass ejections: Observations and simulations. Astrophys J, 2010, 718: 251-265

130 Wild J P, Smerd S F, Weiss A A. Solar bursts. Ann Rev Astron Astrophys, 1963, 1: 291-366

131 Reiner M J, Vourlidas A, Cyr O C St, et al. Constraints on coronal mass ejection dynamics from simultaneous radio and white-light observations. Astrophys J, 2003, 590: 533-546

132 Cho K S, Lee J, Gary D E, et al. Magnetic field strength in the solar corona from type II band splitting. Astrophys J, 2007, 665: 799-804

133 Cho K S, Bong S C, Kim Y H, et al. Low coronal observations of metric type II associated CMEs by MLSO coronameters. Astron Astrophys, 2008, 491: 873-882

134 Robinson R D, Sheridan K V. A study of multiple type II solar radio events. In: Proceedings of Astronomical Society of Australia. 1982, 4: 392-396

135 Shanmugaraju A, Moon Y J, Cho K S, et al. Multiple type II solar radio bursts. Sol Phys, 2005, 232: 87-103

136 Cho K S, Bong S C, Moon Y J, et al. Relationship between multiple type II solar radio bursts and CME observed by STEREO/SECCHI. Astron Astrophys, 2011, 530: A16

137 Mancuso S, Abbo L. Bifurcation of the metric typeII radio emission associated with the giant solar flare of April 2 2001. Astron Astrophys, 2004, 415: 17-20

138 Chen Y, Song H Q, Li B, et al. Streamer waves driven by coronal mass ejections. Astrophys J, 2010, 714: 644-651

139 Chen Y, Feng S W, Li B, et al. A coronal seismological study with streamer waves. Astrophys J, 2011, 728: 147-153

140 Feng S W, Chen Y, Li B, et al. Streamer wave events observed in solar cycle 23. Sol Phys, 2011, 272: 119-136

141 Feng S W, Chen Y, Kong X L, et al. Radio signatures of coronal-mass-ejection-streamer interaction and source diagnostics of type II radio burst. Astrophys J, 2012, 753: 21

142 Kong X L, Chen Y, Li G, et al. A broken solar type II radio burst induced by a coronal shock propagating across the streamer boundary. Astrophys J, 2012, 750: 158

143 Edwin P M, Roberts B. Wave propagation in a magnetically structured atmosphere. Sol Phys, 1982, 76: 239-259

144 Pizzo V, Schwenn R, Marsch E. Determination of the solar wind angular momentum flux from the HELIOS data-an observational test of the Weber and Davis theory. Astrophys J, 1983, 271: 335-354

145 Ginzburg V L, Zhelezniakov V V. On the possible mechanisms of sporadic solar radio emission (radiation in an isotropic plasma). SvA, 1958, 2: 653

146 Dulk G A. Radio emission from the sun and stars. Ann Rev Astron Astrophys, 1985, 23: 169-224

147 Pick M, Vilmer N. Sixty-five years of solar radio astronomy: Flares, coronal mass ejections and Sun Earth connection. Astron Astrophys Rev, 2008, 16: 1-153

148 Cliver E W, Webb D F, Howard R A. On the origin of solar metric type II bursts. Sol Phys, 1999, 187: 89-114

149 Parenti S, Bromage B J I, Poletto G, et al. Characteristics of solar coronal streamers Element abundance, temperature and density from coordinated CDS and UVCS SOHO observations. A\&A, 2000, 363: 800-814

150 Smerd S F, Sheridan K V, Stewart R T. On split-band structure in type II radio bursts from the sun. In: Newkirk G A, ed. IAU Symp. Coronal Disturbances. Dordrecht: Reidel, 1974. 389

151 Vršnak B, Aurass H, Magdalenic J. Band-splitting of coronal and interplanetary type II bursts. Astron Astrophys, 2001, 377: 321-329

152 Saito K. A non-spherical axisymmetric model of the solar K corona of the minimum type. Ann Tokyo Astron Obs, 1970, 12: 53

153 Erickson W C. The Bruny Island radio spectrometer. Publ Astron Soc Austr, 1997, 14: 278-282

154 Howard R A, Moses J D, Vourlidas A, et al. Sun Earth connection coronal and heliospheric investigation (SECCHI). Space Sci Rev, 2008, 136: 67-115

155 Lemen J R, Title A M, Akin D J, et al. The atmospheric imaging assembly (AIA) on the solar dynamics observatory (SDO). Sol Phys, 2012, 275: 17-40

156 Schou J, Scherrer P H, Bush R I. Design and ground calibration of the helioseismic and magnetic imager (HMI) instrument on the solar dynamics observatory (SDO). Sol Phys, 2012, 275: 229-259

157 Li B, Habbal S R, Chen Y J. The period ratio for standing kink and sausage modes in coronal structures with siphon fow. I. Coronal slabs. Astrophys J, 2013(in press)

158 Wang Y M, Sheeley N R, Rich N B. Coronal pseudostreamers. Astrophys J, 2007, 658: 1340-1348

159 Wu S T, Wang A H, Plunkett S P, et al. Evolution of global-scale coronal magnetic field due to magnetic reconnection: The formation of the observed blob motion in the coronal streamer belt. Astrophys J, 2000, 545: 1101-1115

160 Rouillard A P, Lavraud B, Davies J A, et al. Intermittent release of transients in the slow solar wind: 2. In situ evidence. J Geophys Res, 2010, 115: A04104

161 Lin R P, Hudson H S. Non-thermal processes in large solar flares. Sol Phys, 1976, 50: 153-178

162 Lin R P, RHESSI Team, Rhessi. Observations of particle acceleration in solar flares. Adv Space Res, 2003, 32: 1001-1015

163 Feng S W, Chen Y, Kong X L, et al. Diagnostics on the source properties of type II radio burst with spectral bumps. Astrophys J, 2013, doi:10.108810004-637X/766/1/1

164 Zlobec P, Messerotti M, Karlicky M, et al. Fine structures in time profiles of type II bursts at frequencies above $200 \mathrm{MHz}$. Sol Phys, 1993, 144: 373-384

165 Somov B V, Kosugi T. Collisionless reconnection and high-energy particle acceleration in solar flares. Astrophys J, 1997, 485: 859-868

166 Magdalenic J, Vršnak B, Aurass H. Solar type II radio bursts: Emission from shock segments with a collapsing trap geometry? The 10th European Solar Physics Meeting, ESA SP-506, 2002, 1: 335-338

Open Access This article is distributed under the terms of the Creative Commons Attribution License which permits any use, distribution, and reproduction in any medium, provided the original author(s) and source are credited. 